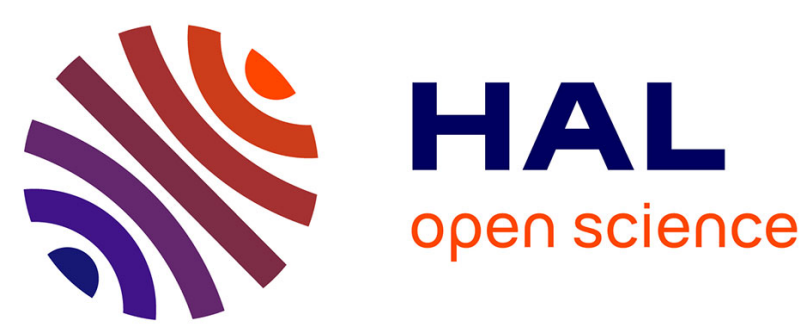

\title{
Evaluating dipolar source localization feasibility from intracerebral SEEG recordings.
}

Vairis Caune, Radu Ranta, Steven Le Cam, Janis Hofmanis, Louis Maillard, Laurent Koessler, Valérie Louis-Dorr

\section{- To cite this version:}

Vairis Caune, Radu Ranta, Steven Le Cam, Janis Hofmanis, Louis Maillard, et al.. Evaluating dipolar source localization feasibility from intracerebral SEEG recordings.. NeuroImage, 2014, 98, pp.118-133. 10.1016/j.neuroimage.2014.04.058 . hal-00988276

\section{HAL Id: hal-00988276 \\ https://hal.science/hal-00988276}

Submitted on 7 May 2014

HAL is a multi-disciplinary open access archive for the deposit and dissemination of scientific research documents, whether they are published or not. The documents may come from teaching and research institutions in France or abroad, or from public or private research centers.
L'archive ouverte pluridisciplinaire HAL, est destinée au dépôt et à la diffusion de documents scientifiques de niveau recherche, publiés ou non, émanant des établissements d'enseignement et de recherche français ou étrangers, des laboratoires publics ou privés. 


\title{
Evaluating dipolar source localization feasibility from intracerebral SEEG recordings
}

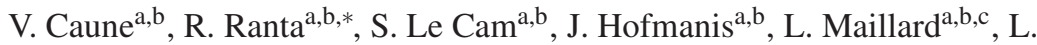 \\ Koessler $^{\mathrm{a}, \mathrm{b}, * *}$, V. Louis-Dorr ${ }^{\mathrm{a}, \mathrm{b}, * *}$ \\ ${ }^{a}$ Université de Lorraine, CRAN, UMR 7039, 54500 Vandoeuvre-lès-Nancy, France \\ ${ }^{b}$ CNRS, CRAN, UMR 7039, France \\ ${ }^{c}$ CHU Nancy, Neurology Department, 54000 Nancy, France
}

\begin{abstract}
Stereo-electroencephalography (SEEG) is considered as the golden standard for exploring targeted structures during pre-surgical evaluation in drug-resistant partial epilepsy. The depth electrodes, inserted in the brain, consist of several collinear measuring contacts (sensors). Clinical routine analysis of SEEG signals is performed on bipolar montage, providing a focal view of the explored structures, thus eliminating activities of distant sources that propagate through the brain volume. We propose in this paper to exploit the common reference SEEG signals. In this case, the volume propagation information is preserved and electrical source localization (ESL) approaches can be proposed. Current ESL approach used to localize and estimate the activity of the neural generators are mainly based on surface EEG/MEG signals, but very few studies exist on real SEEG recordings, and the case of equivalent current dipole source localization has not been explored yet in this context. In this study, we investigate the influence of volume conduction model, spatial configuration of SEEG sensors and level of noise on the ESL accuracy, using a realistic simulation setup. Localizations on real SEEG signals recorded during intracerebral electrical stimulations (ICS, known sources) as well as on epileptic interictal spikes are carried out. Our results show that, under certain conditions, a straightforward approach based on an equivalent current dipole model for the source and on simple analytical volume conduction models yields sufficiently precise solutions (below 10mm) of the localization problem. Thus, electrical source imaging using SEEG signals is a promising tool for distant brain sources investigation and might be used as a complement to routine visual interpretations.
\end{abstract}

Keywords: Electrical source imaging; Stereo-electroencephalography (SEEG); Dipolar source model; Inverse problem; Intracerebral electrical stimulations (ICS)

\footnotetext{
* Corresponding author

** Equal contribution

Email address: radu.rantaduniv-lorraine.fr(R. Ranta)
} 
"NOTICE: this is the author's version of a work that was accepted for publication in NeuroImage. Changes resulting from the publishing process, such as peer review, editing, corrections, structural formatting, and other quality control mechanisms may not be reflected in this document. Changes may have been made to this work since it was submitted for publication. A definitive version was subsequently published in NeuroImage, DOI: 10.1016/j.neuroimage.2014.04.058"

\section{Introduction}

One of the most common techniques for studying the electrical activity of the human brain is electroencephalography (EEG). EEG consists in multichannel recordings acquired using electrodes placed on the surface of the head. These measurements can be used to estimate and localize the underlying brain sources using forward/inverse problem approaches. Forward problem consists in modelling the potentials recorded by the electrodes knowing a source model and a volume conduction model, while the inverse problem aims to retrieve the source knowing the potentials and the volume conduction model. Different approaches exist, none of them being exact because of the bad conditioning of the inverse problem: the number of sources is unknown, the environment model is uncertain and the signals are noisy and sometimes redundant (highly correlated). For very complete reviews of the source localization/estimation problem in EEG the reader is referred to (Michel et al., 2004; Baillet et al., 2001; Greenblatt et al., 2005; Plummer et al., 2008). In epilepsy diagnostics, these techniques are used together with clinical evaluation and medical imaging (CT, MRI, etc.) to assess the location of pathological activities such as epileptic spikes or seizures (Maillard et al., 2009; Koessler et al., 2010) or physiological activity related to cognitive functions (Maillard et al., 2011).

Drug-resistant epileptic patients can also be explored invasively using two different kinds of intra-cranial recordings. The first one is the electrocorticography (ECoG) that consists in placing an electrode array directly on the surface of the brain. It is assumed that the ECoG allows a much better analysis of the sources than the EEG, because of the elimination of the smearing effect of the skull bones and because of a much higher signal to noise ratio (SNR). In the recent literature, rather few source localization attempts based on ECoG analysis were published (Gharib et al., 1995; Zhang et al., 2008; Dümpelmann et al., 2009, 2012; Ramantani et al., 2013).

The second invasive technique is the stereo-electroencephalography (SEEG), consisting in stereotactically placing multi-contact depth electrodes in the brain regions supposed to be responsible for epileptic seizures (Bartolomei et al., 2008; Maillard et al., 2009; Gavaret et al., 2009). In such situation, the local activities of the explored structures are analysed using a bipolar montage (potential differences between two neighbouring contacts/sensors of a depth electrode), which eliminates all propagated activities generated in distant regions, as well as the unknown reference potential. SEEG provides high spatial resolution analysis in the axis of the electrodes, and allows deep, intermediary as well as lateral sources identification in the implanted structures. However, important information might be missed in the unexplored brain volume. Electrical source localization (ESL) approaches using SEEG signals were rarely developed and concerned mainly source localizations of simulated signals (Chang et al., 
2005; von Ellenrieder et al., 2012). Only one study deals with a real application for source localization of temporal auditory areas from intracerebral auditory evoked potentials using distributed source models (Yvert et al., 2005). This method demonstrates a good ability to reproduce the surface evoked potentials when the estimated sources are projected on the scalp electrodes. However, as no ground-truth is available, the accuracy of the method is not further quantified.

Our aim is to extend the role of the SEEG by adapting it to distant dipolar source localization and by analysing the feasibility of this method using simulated and real SEEG signals. The general framework of this study is dipolar localization: in other words, both in simulations and in real recordings, we assume a single dominant source (dipole). This hypothesis has proven useful and accurate for epileptic phenomena (Koessler et al., 2010) and we conjecture that this is also valid for some evoked potentials (Yvert et al., 2005). The current dipole source model was used to represent the dominant activity in the brain also in (von Ellenrieder et al., 2012; Chang et al., 2005; Oosterom, 2012). Source localization using common reference SEEG could be a complementary method to surface EEG based localization. Indeed, one might expect better source localization compared to scalp-EEG source localization, because of the higher signal to noise ratio (no attenuation due to the skull, sensors close to the generators, no extra-cerebral artefacts) and to the less complex volume conduction model. The authors would like to mention that a preliminary version of this work was presented in (Caune et al., 2013).

We focus on three main issues influencing the localization performances: the propagation model (i), the spatial distribution of the measuring contacts (ii) and the effect of the noise and/or disturbing activities (iii). Indeed, although Finite Element Models (FEM) models are known to provide realistic, high quality and subject-specific head propagation models, their application requires a precise segmentation of the different head tissues as well as a realistic estimation of their electrical conductivities, and is therefore highly computationally demanding. For these reasons, one can be reluctant in developing such models to solve the ESL problem and might turn to simpler analytical approaches such as the infinite homogeneous model (IHM) or the spherical models (one-sphere-OSM, or multi-sphere-MSM). The second important point that we aim to analyse is the impact of the spatial distribution of the measuring contacts (and thus of the depth electrodes), which have a higher irregular spatial sampling compared to the surface EEG. Should all available contacts be used? How many of them are necessary for reliable results? Is it sufficient to implant only one hemisphere? What can we expect as localization result when the dipole to be localized is placed in a non-implanted hemisphere? Finally, a last addressed point is the effect of the noise and/or of the background activities on the localization performances (i.e. assuming that the dipole to be localized is not unique and there are other active regions in the brain). These studies (model accuracy, sensor conditioning and noise/nuisance influence) are conducted in a realistic simulation setup (individual head model without anatomical abnormalities), and are finally assessed (i) on real SEEG signals recorded during clinical electrical intra-cortical stimulations (ICS), thus containing a known dipolar source and (ii) on an example of interictal epileptic period with spikes. 


\section{Material and Methods}

\subsection{SEEG signals and sources}

\subsubsection{General considerations}

The considered measurements are issued from SEEG recordings, using depth electrodes placed directly into the brain tissue. Every electrode has from 5 to 15 collinear measuring contacts having a length of $2 \mathrm{~mm}$ and separated by $1.5 \mathrm{~mm}$ of isolating matter (3.5 mm between their centres). The diameter of an electrode is of $0.8 \mathrm{~mm}$ (DIXI Microtechniques, Besançon, France). Unlike for surface EEG, the electrodes (thus the sensors) are not distributed over the whole (or most of) cortical area and there is no standardized spatial placement procedure. Therefore, when aiming to use SEEG measurements for source localization, it is important to take into account this particular placement.

The simplest recording setup for the electrical source localization could be one multi-contact depth electrode. The sensors are in this case collinear and the localization problem is undetermined, regardless of the number of available signals. Consequently, at least two depth electrodes are needed. Moreover, they have to be in different planes. Indeed, a symmetrically placed dipole (with respect to a plane) will produce the same potentials on coplanar sensors. Besides, dipoles having their origin in the plane are also undetermined (the orthogonal component is invisible to the sensors). Although this situation is hypothetical, as the electrodes are seldom inserted in the same plane, the noise (i.e. the background activity) can mask the dipole of interest components on sensors situated in a sort of slice of brain tissue, a kind of thicker version of a plane.

A dipolar source is determined by 6 parameters: three for the position and three for the orientation and amplitude. Consequently, at least 6 measurements are needed to perform the localization task. In our simulations, as well as for real examples, we considered thus at least 6 contacts placed on 3 or more depth electrodes. We also took the precaution of not considering two contiguous sensor measurements of a same electrode, in order to get a good disparity on the propagation of the equivalent dipole to be located. More precisely, four spatial configurations for the sensors were analysed:

(i) a subgroup of 6 well chosen sensors, i.e. the 6 sensors having the maximum absolute potential, from at least 3 different electrodes and not contiguous on a given electrode. We would like to assess whether these 6 selected (hopefully well conditioned) measurements are enough to provide good localization results,

(ii) all the sensors in the hemisphere of the dipole (ipsilateral), knowing that it can be well implanted or not. When using real recordings (see below subsection 2.1.3), the sensors from the ICS electrode and those recording the highest amplitude epileptic spikes for the real interictal SEEG, are not considered,

(iii) all the sensors in the opposite hemisphere of the dipole (contralateral). Again, the influence of the number of sensors will be analysed.

and (iv) the whole set of available sensors inside the brain (the same electrodes were excluded as for the previous ipsilateral-sensors configuration)

From a signal quality point of view, SEEG signals avoid noise, physiological artefacts (especially muscular activity) and the attenuating effect of the skull. As the sensors are placed closer to the generators, the signal to noise ratio (SNR) is generally high, especially if the reference contact is almost electrically inactive. Still, in real 
recordings, if the site of the reference electrode is active, the SNR will be affected, which may impact the accuracy of source localization unless techniques are used to account for this problem (e.g., signal re-referencing). Depending on the application and on the measurement setup, one can for example average in time (for repetitive pattern localization), average in space like in surface EEG (Pascual-Marqui, 2007) (although, as the sensors are not homogeneously distributed in all the brain, the reference cannot be realistically eliminated by averaging) or by some weighted average based on statistical hypothesis (Hu et al., 2008; Ranta et al., 2010; Madhu et al., 2012). In this paper we assume a zero reference signal in the simulation case and we adopt the first solution above (time averaging) for the real signals. This allows to assess the localization performances with respect to the announced criteria (head model, SEEG sensors positions, SNR of the SEEG sensors) and discard the reference influence.

\subsubsection{Simulation setup}

Ideal no-noise case. As starting hypothesis, we assume a single active dipolar source inside a realistic head model (5 volumes, i.e., scalp, skull, CSL, grey and white matter, issued from an automatic Freesurfer segmentation procedure). The following simulation procedure is adopted: the head volume of a patient without anatomical malformation is chosen. We also consider a realistic placement for the simulated SEEG electrodes, based on an implantation scheme commonly performed in temporal lobe epilepsy context. More precisely, 9 simulated electrodes were positioned in the right hemisphere and 3 in the left one, each one having between 7 and 10 equally spaced contacts inside the brain. A total of 112 sensors are implanted, 86 in the right hemisphere and 26 in the left hemisphere. A realistic lead-field matrix was obtained by FEM modelling, as described in section 2.2.

In the absence of noise, the localization results depend on the simplified head model employed for solving the inverse problem and on the relative position of the dipole to be localized with respect to the set of used sensors. In order to obtain a complete view, dipolar sources positions were fixed on a regular 3D grid having $9 \mathrm{~mm}$ between points, covering the whole brain volume. Next, only those situated inside the segmented gray matter were kept, which led to 506 possible source positions. For every position, we considered the three orthogonal orientations ( $O x$ : inion-nasion, $O y$ : right-left and $O z$ : bottom-up).

A second objective is to evaluate the robustness of the method in presence of noise and disruptive sources, i.e., when the hypothesis of the single source is relaxed. Indeed in real SEEG, it is highly unlikely to observe (only) one source all over the electrodes: either background or parasite sources will degrade the SNR, especially on distant electrodes, where the source of interest is strongly attenuated. We thus propose the two following configurations on simulation:

Independent background activity. A first simplified noisy model is an additive, temporally and spatially white Gaussian noise added to the simulated measurements, (aiming to model independent background activity). One hundred noise realizations were simulated per dipole location and orientation. The huge amount of time needed to perform these simulations prevents us from carrying these experiments for the whole set of the 506 dipoles. We have then chosen a subset of 50 dipoles, selected according 
to two criteria: (i) they have to be distributed within the whole brain volume and (ii) they have to be well localized in the absence of noise.

Identical white noise levels are added on each measurement, hence the same noisy context whatever the position and the orientation of the dipoles to be localized. Two noise levels are used, corresponding to standard deviations $\sigma_{1}$ and $\sigma_{2}$. These noise levels are computed with respect to the mean level of the simulated "clean" activity $M_{p}$ measured by all electrodes. More precisely, $\sigma_{1}=M_{p} / 10$ and $\sigma_{2}=M_{p} / \sqrt{2}$ (i.e., a noise attenuated by $20 \mathrm{~dB}$, respectively $3 \mathrm{~dB}$, with respect to $M_{p}$ ), where

$$
M_{p}=\frac{1}{M \cdot D} \sum_{d=1}^{D} \sum_{m=1}^{M}\left|V_{d m}\right|,
$$

is the mean magnitude of the potentials $V_{d m}$ (generated by dipole $d$ on electrode $m$ ) over the whole set of $M=112$ sensors and the $D=50$ considered dipoles.

In practice, in our simulation, the noise having a standard-deviation $\sigma_{1}$ is lower than the potentials generated by a dipole up to a distance of about $15 \mathrm{~cm}$ in the direction of the dipole, while this distance reduces to about $5 \mathrm{~cm}$ for the noisier simulation $\left(\sigma_{2}\right)$. In other words, we might say that the $\sigma_{1}$ noise level corresponds to a source visible almost all over the brain, while the $\sigma_{2}$ noise models a source mainly visible on neighbouring electrodes. It has to be emphasized that the SNR of a particular measurement will also be function of the dipole orientation relatively to the sensor (e.g. for a given distance, a supplementary $3 \mathrm{~dB}$ attenuation appears with an angle of $45^{\circ}$ ).

It has to be noticed that the time averaging also has an impact on the SNR of the signals used for localization. Indeed, a common technique to improve the SNR is to average similar patterns (in general spikes or evoked potentials) over several trials. In the white noise case, if this number of trials is infinite, we theoretically get an infinite SNR. Within this context, the $\sigma_{1}$ noise level stands for a good time averaging (although we must note here that identifying patterns to be averaged on spontaneous EEG is not trivial, see also the clinical example presented in the Results section), while a noise with $\sigma_{2}$ standard-deviation approximates a case where the activity is weak and/or few time instants are available for averaging.

We computed the position error for each of the 50 dipoles and for the four sensor configurations using 100 noise realizations for each noise level. The 100 position errors per dipole and noise level were next averaged and the standard deviation was computed. We have thus obtained 50 average position errors and their standard deviations per noise level for each sensor configuration, thus 1200 couples of values (50 dipoles, 4 sensor configurations, 2 noise levels, 3 orientations). These values reflect how the method is expected to perform for different dipoles positions in the brain volume. In order to give a synthetic view of these performances (regardless of each dipole specific position and orientation), the error values are averaged over the orientations $\mathrm{Ox}, \mathrm{Oy}, \mathrm{Oz}$. Next, the medians of these 50 average errors are computed, indifferently of the position of the dipole (whole head volume), but also with respect to the left and right hemispheres ${ }^{1}$.

\footnotetext{
${ }^{1}$ The left hemisphere contains 20 dipoles while the right hemisphere contains 30 dipoles.
} 
Source of nuisance. A second disruptive scenario involves one or more parasite sources situated in other brain regions than the dipole to be localized. It is of course impossible to take into account all the possible combinations. We have therefore simulated some simple illustrative examples, considering three source placements: the source $D 1$ is placed in the deep structures within the hippocampus, while the source $D 2$ is placed in a gyrus in the intermediary structures and the source D3 is located on the brain surface near the skull, in the lateral temporal cortex. The perturbing dipole was placed in the middle of the contralateral hemisphere. The direction of the dipole to be localized and of the perturbing one were either parallel (i.e., along $O x$ or $O z$ ) or they were both pointing towards the opposite hemisphere (thus along $O y$ ). The amplitude of the nuisance was simulated according to an almost similar procedure as for the white noise: either divided by $10\left(\sigma_{1}\right)$ or by $\sqrt{2}\left(\sigma_{2}\right)$.

\subsubsection{Real recordings: Intra-cerebral stimulation (ICS)}

Because of its invasiveness, SEEG is dedicated, in humans, to difficult cases of drug-resistant epileptic patients. Ten to fifteen depth electrodes, each one having 5-15 equally spaced measuring contacts are placed within the selected brain areas in order to localize and delineate the epileptogenic zone. Locations of the depth electrodes differ from patient to patient because of different electroclinical hypothesis. Prior comprehensive evaluation include detailed medical history and neurological examination, neuropsychological testing, high resolution magnetic resonance imaging (MRI), interictal and ictal (when available) single photon emission computed tomography (SPECT), positron emission tomography (PET), and high resolution long-term scalp EEG including ictal recordings (Maillard et al., 2009).

This study includes one 40-year-old man with presumed bitemporal lobe epilepsy. Usual seizures started with bilateral auditive hallucinations (wind sound). No anatomical lesion was found in MR images. The patient gave his informed consent prior to participation. According to the electroclinical hypothesis, he was implanted with ten depth electrodes in the right temporal lobe and insular cortex and four in the left temporal lobe. The reference was FPz surface electrode from the classical 10-20 system. The depth sensors coordinates were automatically determined using the procedure described in Hofmanis et al. (2011).

The known sources, used to assess the localization performances, were in-vivo intracerebral stimulations (ICS) used in clinical routine. The aims of the ICS during the SEEG investigations are (i) to identify the epileptogenic cortical structures whose stimulation elicits the usual seizures and (ii) to evaluate the residual cognitive function of these implanted structures (Jonas et al., 2012, 2014). In our source localization context, the ICS signal can be seen as a dipolar generator of electrical activity artificially placed at a known location and orientation inside the brain. ICS were applied between two contiguous contacts along one common depth electrode. Bipolar ICS were performed at $53 \mathrm{~Hz}$ during 5 seconds with a constant amplitude of 1 or $1.2 \mathrm{~mA}$, depending on the stimulation site.

Before applying the localization procedure on the ICS signals, some basic preprocessing steps need to be performed. A window of 2.5 seconds was chosen during 
the stabilized stimulation period ${ }^{2}$. A simple high-pass filter followed by thresholding was applied to detect the peaks of the stimulation patterns. Between 60 and 90 peaks were detected during the 2.5 seconds (below the maximum value of 132 peaks corresponding to a stimulation frequency of $53 \mathrm{~Hz}$ ). Their amplitudes were averaged electrode by electrode to obtain the amplitudes of an averaged ICS pattern, used further in the localization procedure. Despite this preprocessing, we must underline that there is no guarantee that the physiological activity recorded by other sensors will be eliminated by averaging (although white noise will be substantially reduced). Finally, the contacts belonging to the electrodes generating the ICS has been discarded from the localization procedure, the contacts close from the stimulation site being saturated by the strong electrical field. Also, we would like to assess if such strong dipole could be localized using only far measurements.

Three different sites of ICS stimulations, from respectively deep to superficial brain localizations, were chosen in this study: TB'2-3 situated in the entorhinal cortex, TB'45 in the perirhinal cortex and TB' $8-9$ in the inferior temporal gyrus. All of them were in the left hemisphere, less implanted than the right one. As for the simulated signals, we used the four spatial sensors configuration described above (subsection 2.1.1).

\subsubsection{Real recordings: Interictal epileptic spikes}

A 28 year-old woman with drug-resistant insulo-opercular epilepsy was included in this study. She gave her informed consent prior to participation. Epileptic seizures started with a left-side head deviation and a right upper limb tonic elevation. After presurgical evaluation, depth EEG recording was performed using ten depth electrodes implanted in the insulo-opercular regions as follow (internal and lateral contacts): P', cingulum/parietal operculum ; T', infero-anterior insula/ superior temporal gyrus ; B', anterior insula/pre-motor cortex; $X^{\prime}$, posterior insula/post-central gyrus; F', and L', anterior and posterior part of the inferior frontal sulcus/middle frontal gyrus; R', middle insula/central operculum; C', cingulum/middle frontal gyrus; S', superior frontal sulcus; M', supplementary motor area/superior frontal gyrus. During SEEG investigation, interictal epileptic spikes were recorded by the R' depth electrode. These spikes were selected for this study due to (i) their relatively high signal to noise ratio and (ii) their focal localization, i.e., the absence of other co-activated epileptic sources (Figure 8, left side).

The coordinates of the depth sensors were obtained as for the ICS localization described above. Time averaging was equally performed using a similar procedure: the signal from the R' 6 contact (presenting highest amplitude spikes with no co-activated source in the other contacts) was high-pass filtered and thresholded. Twenty spikes were selected by the procedure and confirmed by a trained electrophysiologist. One hundred time samples belonging to these 20 spikes overpassed the chosen threshold (set at one half of the amplitude of the highest spike) and thus averaged.

\footnotetext{
${ }^{2}$ It has to be noted that the contacts used for stimulation were used, before and after the stimulation period, as recording contacts. Therefore, an electrical commutation artefact lasting up to 2 seconds might appear at the beginning of the ICS period (see also Hofmanis et al. (2013) for more details).
} 


\subsection{Forward problem and head volume modelling}

Given the frequency range of the brain activities and taking into account the distances between sources and sensors, a common assumption that we follow is that the mixing is instantaneous, i.e. linear. Consequently, at a given time instant, the potentials recorded by the electrodes can be written as:

$$
\boldsymbol{\Phi}_{0}=\mathbf{K} \cdot \mathbf{J}
$$

where $\mathbf{K} \in \mathbb{R}^{N_{c} \times\left(3 N_{s}\right)}$ is the lead field matrix corresponding to the $N_{s}$ sources responsible for the electromagnetic activity on the $N_{c}$ sensors and $\mathbf{J} \in \mathbb{R}^{\left(3 N_{s}\right) \times 1}$ is the current density vector (in three directions $O x, O y$ and $O z$ ) for the $N_{s}$ sources.

Different approaches exist to compute the environment model in $K$, from simple analytical models to realistic anatomical models computed numerically for each patient. Our first aim is to evaluate respectively the simple infinite homogeneous medium (IHM), as well as the One-Sphere Model (OSM), in comparison to the realistic highly demanding FEM. Indeed, one might reasonably suppose that the SEEG measurements are taken inside the brain volume, which is often modelled as homogeneous and isotropic. The IHM and the OSM might then be eligible to perform accurate brain sources reconstruction from SEEG. Such hypothesis was already made in (Chang et al., 2005) and tested in (Cosandier-Rimélé et al., 2007). We propose here further experiments and comparisons to make our arguments more convincing.

\subsubsection{Finite Element Modelling}

The most elaborate approach, considered here as a good approximation of real heads, is proposed by Finite Element Models (FEM), which aim to model as close as possible the real individual anatomy and the conductivities properties. FEM are able to treat irregular shapes, extracted from imaging techniques, as well as inhomogeneous and anisotropic environments, by assigning different conductivity values to each matter (tissues) and (for anisotropic models) for each direction.

Forward problem using the FEM method starts with the segmentation of the head tissues obtained by MRI and CT imaging techniques. Five compartments are segmented in our case: gray matter, white matter, cerebrospinal fluid (CSL), bone and scalp. The second step includes the generation of a volume mesh that represents geometric properties of the volume. In our particular setup, additional mesh points were added at the coordinates of the centre of each SEEG contact. The number of resulting tetrahedrons was 2009270, for a number of node points (DoF) of 323065. The shortest edge is $0.04 \mathrm{~mm}$ and the longest one is $5.15 \mathrm{~mm}^{3}$. In this paper, the conductivities were chosen constant for a given tissue, regardless of the orientation $(0.33 \mathrm{~S} / \mathrm{m}$ for the grey matter, $0.2 \mathrm{~S} / \mathrm{m}$ for the white matter, $0.33 \mathrm{~S} / \mathrm{m}$ for the scalp and $0.004 \mathrm{~S} / \mathrm{m}$ for the skull bones (Geddes and Baker, 1967)). Discretized version of the Poisson equation was used to compute the potentials in every point of the mesh and/or for every electrode (see (Vallaghé, 2008; Hofmanis, 2013) for a detailed description). Following

\footnotetext{
${ }^{3}$ We have to mention that the mesh used in all the figures in this paper is a coarse version of the mesh used in simulations.
} 
(von Ellenrieder et al., 2012), we have chosen to approximate the SEEG contacts by their central points, as the influence of the electrode dimension on the localization was proven to be very limited.

All the forward problems simulated in this paper, i.e., for all the artificial sources described in section 2.1.2, are generated using this numerical model. On a modern PC, the complete FEM computation time in Matlab for one dipole is about 10s. For our grid of 506 tested dipoles having three orientations, it takes more than 4 hours.

\subsubsection{Analytical models}

Our starting hypothesis is that simple analytical models such as IHM and OSM are sufficient for accurate localization in the SEEG context.

The main advantage of the IHM is its fast calculation time. It is assumed to predict fairly well the measurements inside the brain, provided that the hypothesis of homogeneity and isotropy are valid. In this case, the $3 \mathrm{D}$ row vector $k_{i j}$ (one element of $\mathbf{K}$ in eq. 2) writes:

$$
k_{i j}=\frac{1}{4 \pi \sigma} \frac{\left(r_{\phi_{i}}-r_{j}\right)}{\left\|r_{\phi_{i}}-r_{j}\right\|^{3}}
$$

where $\sigma$ is the conductivity of the medium, $r_{\phi_{i}}, r_{j} \in \mathbb{R}^{1 \times 3}$ are position vectors for the $\mathrm{i}$-th electrode and for the $\mathrm{j}$-th source respectively and $\|$.$\| designates the L2-norm. Of$ course, these relations can be used to compute the potentials in every chosen point of the $3 \mathrm{D}$ space.

The OSM is also based on an assumption of homogeneity and isotropy in the brain volume. This volume is fitted within a sphere roughly modelling the bound between the skull and the brain, thus taking account of the difference in conductivity between inside and outside the brain. This model remains analytically tractable and the equations can be found in (Yao, 2000).

For the IHM as well as for the OSM, we consider the brain conductivity $\sigma=$ $0.33 \mathrm{~S} / \mathrm{m}$.

\subsubsection{Forward model comparisons}

The comparisons of the forward solutions based on the two analytic models (IHM and OSM) with the one provided by the FEM model are carried out considering three examples of source placements: the source $S 1$ is placed in the hippocampus, the source $S 2$ is placed in a gyrus in the intermediary structures and the source $S 3$ is located on the brain surface near the skull. For each of these three placements, the three orthogonal orientations $(\mathrm{Ox}, \mathrm{Oy}, \mathrm{Oz})$ are considered in order to evaluate the influence of the tissue limits and of the skull border considering each possible orientation components. The electrical fields generated by these three dipoles (thus nine configurations) are computed on each vertex of the head volume mesh using the three considered propagation models. Maps of difference are then computed between IHM and FEM as well as between the OSM and FEM, using the following error measurement:

$$
\Phi_{e}=\left|\Phi_{F E M}-\Phi_{a}\right| / \Phi_{F E M}
$$

$\Phi_{a}$ being the potential values computed either using IHM or the OSM. 


\subsection{Inverse problem}

\subsubsection{Problem Statement}

Two main classes of inverse problems are to be distinguished: the under-determined case where a dipole is placed onto each mesh node of the volume with fixed orientation (orthogonal to the brain surface), resulting in distributed source models whose amplitudes are estimated using source imaging techniques (see (Baillet et al., 2001) for a review); and the over-determined case when few dipoles are considered $\left(6 * N_{s} \ll N_{c}\right)$, leading to parametric (or dipolar) approaches (Scherg, 1990; Kiebel et al., 2008). This work deals with this second class of methods. In specific cases (high amplitude interictal spikes for example), one of the brain regions (sources) generates a signal having a much higher amplitude than the other regions. In this case, the recorded electrical activity can be approximated with one equivalent dipole and we are in the over-determined case, thus looking for an optimal solution. We focus in this research on this particular model. To better understand the influence of the different parameters on the results and assuming one main source of interest, we rewrite the mixing model (2) as:

$$
\boldsymbol{\Phi}(t)=\mathbf{k}_{1} \cdot \mathbf{j}+\mathbf{N}
$$

where $\mathbf{j}$ is a vector containing the projections of the dipole on the 3 axes, $\mathbf{k}_{1}$ is the vector corresponding to the projections of $\mathbf{j}$ on the sensors $\left(N_{c} \times 3\right)$, and $\mathbf{N}$ contains the projection of all the other sources on the electrodes, seen here as additive noise (from the main dipole point of view).

As the problem is non-linear and over-determined, the optimal solution is generally obtained by iterative non-linear optimisation techniques. In this paper we used a fixed dipole approach, implemented as a simplified version of the algorithm proposed by (Scherg, 1990). This algorithm is briefly described in the following.

\subsubsection{Fixdip algorithm}

The main difference between an instantaneous dipole localization (moving dipole) and the Scherg's method is the introduction of the time index. In this case, (5) can be rewritten as:

$$
\boldsymbol{\Phi}(t)=\mathbf{C}_{1} \cdot s_{1}(t)+\mathbf{N}(t)
$$

where $\mathbf{C}_{1}$ is a gain vector determined by the position and the orientation parameters of the dipole to be estimated, the electrode positions and the chosen forward model, while $s_{1}$ stands for the dipole amplitude.

Next, an initial position and orientation are chosen and an initial estimate gain vector $\hat{\mathbf{C}}_{1}$ is computed using one of the forward models described above (in our case IHM or OSM). A first estimate of $s_{1}(t)$ is obtained as the least squared error solution of the over-determined system (6):

$$
\hat{s}_{1}(t)=\hat{\mathbf{C}}_{1}^{\dagger} \cdot \boldsymbol{\Phi}(t)
$$

where $\hat{\mathbf{C}}_{1}^{\dagger}$ denotes the Moore-Penrose pseudo-inverse of $\hat{\mathbf{C}}_{1}$. The residual error, obtained as:

$$
R V=\sum_{t}\left\|\boldsymbol{\Phi}(t)-\hat{\mathbf{C}}_{1} \cdot \hat{s}_{1}(t)\right\|^{2}
$$


is next minimized by optimizing $\hat{\mathbf{C}}_{1}$ using a simplex algorithm in 5D (the three spatial coordinates and the two angles defining the orientation of the dipole $\mathbf{j}_{1}$ ). Once the optimum is reached, a new value of $\hat{s}_{1}(t)$ is estimated by (7) and the procedure is iterated until convergence.

Considering time evolution of the amplitude and of the noise allows in principle to better estimate the dipole characteristics (assumed to represent time varying activations of a given population of neurons) and, most of all, to somehow average the influence of the uncorrelated background activity $\mathbf{N}(t)$. In this paper, we deal with fixed dipoles of fixed amplitude. This is equivalent, in simulation, to considering a single time instant so dropping $(t)$ again. On real signals, the ICS dipole has a repetitive pattern, with a constant peak amplitude (and obviously a constant position and orientation). Two options are then possible: (i) using the Scherg's algorithm as described above after choosing the peaks of the stimulation as time indices $t$ or (ii) averaging several peaks and localize this "average" pattern (with no time index, as in the simulation case). We have tested both approaches and the results are almost equivalent, with a slight advantage for the second option. The Results section presents therefore only this case.

Finally, one has to remind that the optimization procedure might converge to a local minimum. This observation enlightens the role of the initialization point. Indeed, in practical applications, the results vary significantly depending on the initialization. In order to automatize the localization procedure, we propose a multi-start algorithm, implemented as follows: (i) generate equidistant initialization points on a cubic grid (in our case 27 points); (ii) keep the points situated inside the sphere fitted for the OSM (19 in our case, the 8 corners of the cube being outside the sphere; the minimum distance between the initialization points is about $4 \mathrm{~cm}$ ) (iii) perform independent localizations, using the fixdip algorithm described above, for each initialization and (iv) chose the solution having the minimum residual variance $R V$ (8), i.e., the maximum goodness of fit:

$$
G O F=100 \frac{\|\boldsymbol{\Phi}\|^{2}-R V}{\|\boldsymbol{\Phi}\|^{2}}
$$

Although the use of this criterion might seem natural, the GOF is not always reflecting good localization performances. More details on this point will be presented in the Results and Discussion sections.

We perform the described localization procedure separately on the 506 simulated dipoles placed all over the gray matter of the same subject. For each dipole, we consider the 3 possible orientations (along $O x, O y$ and $O z$ ). These dipoles are propagated one by one on the simulated SEEG sensors using the FEM model, thus yielding $3 \times 506=$ 1518 distinct localization problems, and are localized using the two analytical models (IHM and OSM). The 4 different configurations of sensor placement described in the section 2.1.1 are used to perform the localization task.

\section{Results}

This section is structured in three main parts: the first one briefly evaluates the forward models involved in this research (IHM and OSM) versus a realistic FEM model, considered as ground-truth. Next, the inverse problem localization performances are 
evaluated on the simulated configurations described in the section 2.1, using both the IHM and the OSM for the inversion. Finally, the localization method is applied and evaluated on real SEEG recordings. Two types of signals are presented: SEEG recorded during ICS, thus in the case of a known generator, and SEEG recorded during a sequence of epileptic spikes.

\subsection{Environment model comparisons}

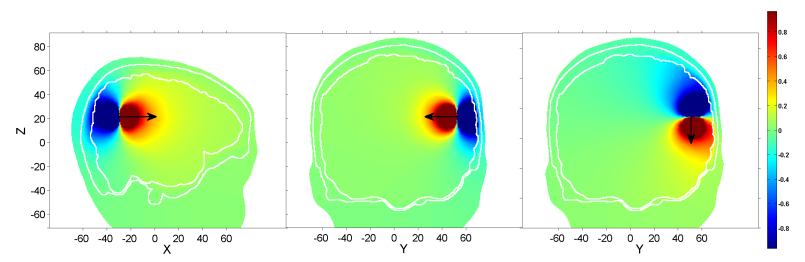

Figure 1: The lateral dipole $S 3$ propagation using FEM. All orientations $(O x, O y, O z$, from left to right). The dipole is represented as a thin black arrow. Axes units: $\mathrm{mm}$.

We compare the two analytical models with the realistic FEM using the error defined in (4). The propagation using the FEM model is given for the dipole $S 3$ on figure 1. The colors (corresponding to potential values) on this figure are saturated at $10 \%$ of the potential computed by the IHM at a $5 \mathrm{~mm}$ distance of the dipole origin, in the direction of the dipole. This value is very similar to the one given by the FEM (see figure 2) and it was chosen in order to have a similar color code regardless of the position and of the orientation of the dipole. Saturating the image allows to highlight (in dark red/ blue) the brain volumes where the potentials have important magnitudes and thus where the comparison with the analytical models is relevant (the error of the analytical models should be small). Outside this area, we can observe the decrease of the potential, roughly with the square of the distance (Zaveri et al., 2009), yielding small potential values ( $10 \%$ of the saturation value, i.e., 100 times smaller than the potential at $5 \mathrm{~mm}$ from the dipole, green color) at about $5 \mathrm{~cm}$ from the origin of the dipole.

The error $\Phi_{e}$ is illustrated on brain slices in the dipole plane. The results for the three dipoles $S 1, S 2, S 3$ are provided in the figure 2. For the sake of visualization, the error value is saturated to 1: dark red areas indicate errors of more than $100 \%$ with respect to the FEM model.

From the error maps of the profound dipole $S 1$, we can see that both the OSM and even the IHM provide satisfactory approximations of the realistic FEM propagation model in the middle of the brain volume, at least near the dipole placement, with a correct advantage for the OSM. In this area are concentrated most of the SEEG sensors, thus being a first positive argument toward a localization procedure based on these simple models. It should be noted that high errors are encountered in the vicinity of the plane orthogonal to the dipoles, the electrical field values being very close to zero in this area, regardless of the model. Also, when interpreting these maps, one must have in mind that high relative errors encountered far from the dipole are rather irrelevant, because the electrical field is also very close to zero regardless of the model. 


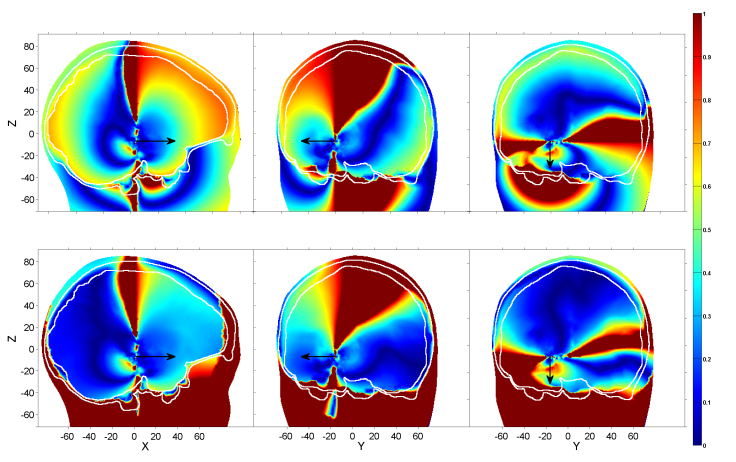

(a) Profound dipole $S 1$

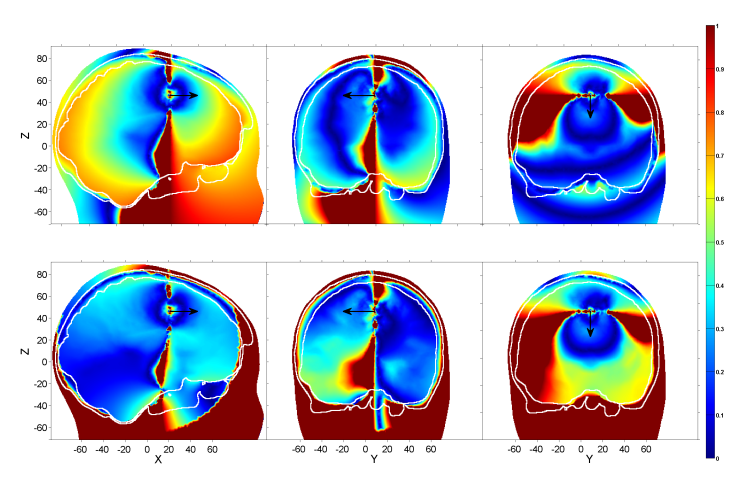

(b) Intermediary dipole $S 2$

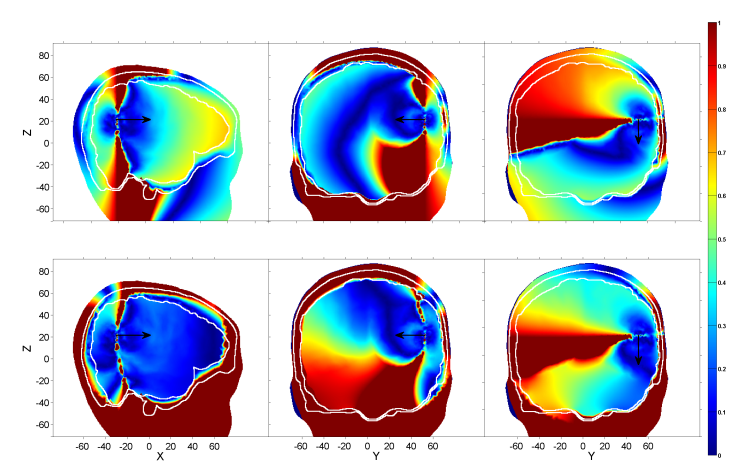

(c) Superficial dipole $S 3$

Figure 2: Difference maps for the three dipoles $S 1, S 2, S 3$. For each subfigure, the first row illustrates the difference IHM vs FEM, while the second one the difference OSM vs FEM. From left to right, the three standard orientations of the dipoles, represented as thin black arrows: $O x, O y, O z$. Axes units: mm. 
The results for the two other dipoles confirm the previous analysis, even if they show slightly less advantages for the OSM: for some positions and orientations, the IHM seems to provide better approximations of the electrical potentials in deep structures, while the OSM looks more accurate near the skull border, if the sphere is correctly fitted (see dipole $S 3$, placed near to the skull border).

From these observations, we expect reasonably good localization performances using these simple analytical models, in particular when considering deep and intermediate source placements. Better results should be provided by the one-sphere model. These results also confirm that the localization of sources placed near the skull border might be poorer. The preliminary conclusions made here have to be tempered by the fact that the referenced we used, namely the FEM model, still remains an approximation of the propagated field, and also includes its own modelling errors. This evaluation simply points out that we can be as much confident in analytical models than in FEM when considering particular areas in the middle and deep brain volume, such areas coinciding with most of the SEEG sensor positions.

\subsection{Localization results on simulated signals}

\subsubsection{Ideal no noise case}

The localization results using the IHM and the one sphere model are provided in the table 1. The median of the error distances are given for each of the two models, the four configurations, and the three orthogonal orientations $O x, O y$ and $O z$. Also, we provide the results by hemisphere so that the influence of the number of sensors can be analysed. At a first glance, it can be seen that the one sphere model outperforms the IHM one. We then give more details on the one sphere model inversion results as boxplots in the fig. 3 , for each of the four configurations and for both left and right hemispheres. We will consider that a satisfactory localization performance corresponds to a position error of less than $1 \mathrm{~cm}$, a margin which is usually considered relevant in clinical application. Figure 4 illustrates the maximum and mean OSM-based localization errors (over the orientations $\mathrm{Ox}, \mathrm{Oy}, \mathrm{Oz}$ ) for each sensor configuration, for 8 brain slices (transversal planes $y O z$ ). The position errors for the dipoles are given using a discrete color code (see legend).

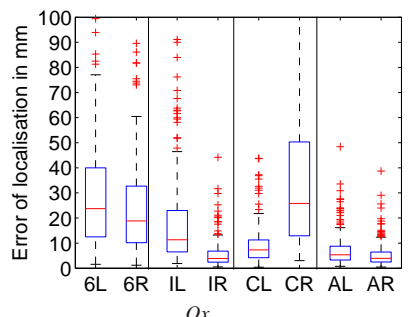

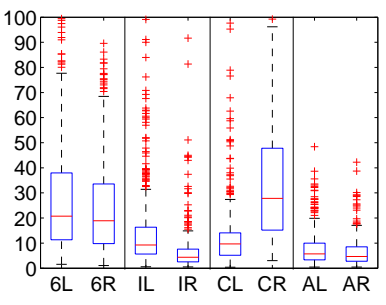

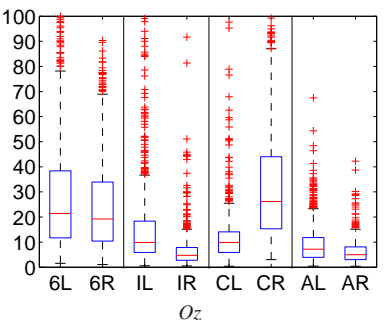

Figure 3: Boxplots of the OSM-based localization performance for the three dipole orientations. The results are presented for four sensor configurations (6 highest absolute values (6), Ipsilateral (I), Contralateral (C), and All (A)) and for each hemisphere (left (L), right (R)) 


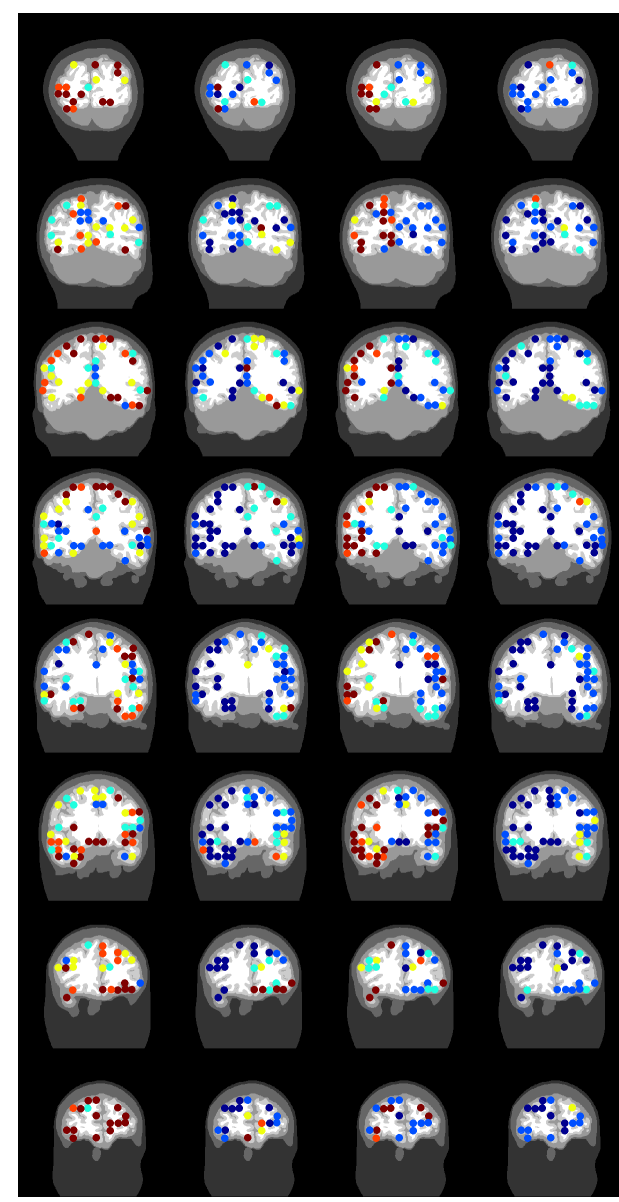

(a)

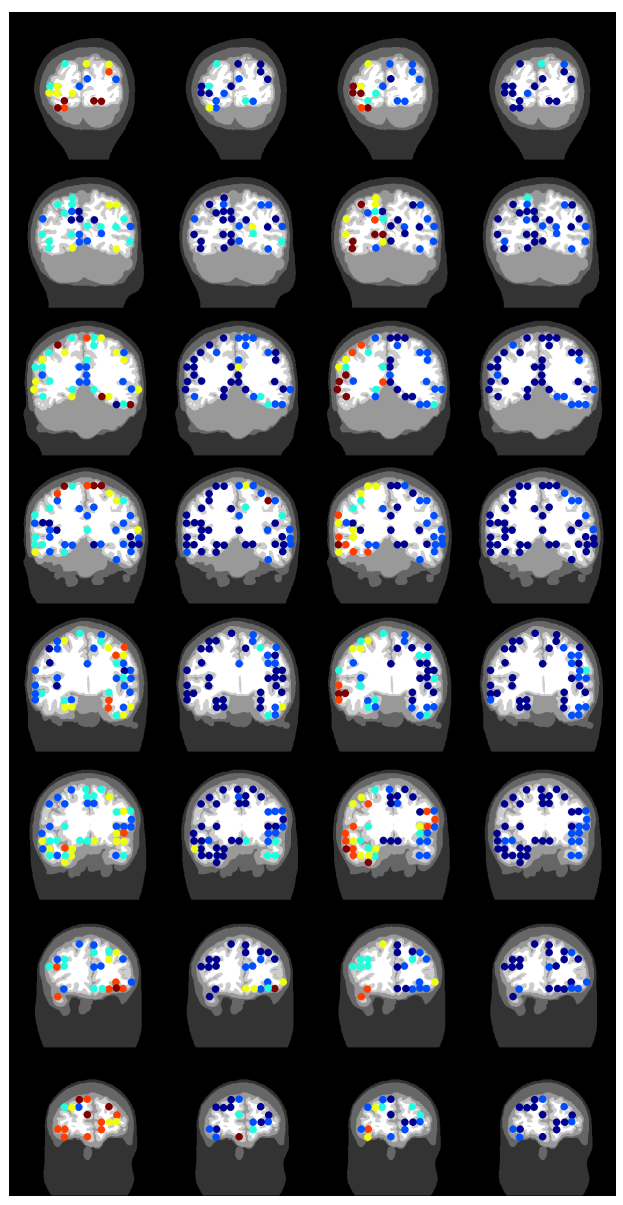

(b)

Figure 4: Maximum (a) and mean (b) of the localization errors (using the one-sphere model) over the orientation $(O x, O y, O z)$ for the 506 dipoles regularly distributed in the grey matter, given for the 6 sensors (first column), the ipsilateral sensors (second column), the contralateral sensors (third column), and the all-sensors (fourth column) configurations. The results are given for 8 brain slices in the transversal planes $y \mathrm{Oz}$, from the back to the front head (i.e., the right hemisphere is figured on left sides). Position error color code: $\bullet \varepsilon_{p} \in\left[0,1\left[\mathrm{~cm}, \bullet \varepsilon_{p} \in\left[1,2\left[\mathrm{~cm}, \bullet \varepsilon_{p} \in\left[2,3\left[\mathrm{~cm}, \bullet \varepsilon_{p} \in\left[3,4\left[\mathrm{~cm}, \bullet \varepsilon_{p} \in\left[4,5\left[\mathrm{~cm}, \bullet \varepsilon_{p} \geq 5 \mathrm{~cm}\right.\right.\right.\right.\right.\right.\right.\right.\right.\right.$ 


\begin{tabular}{|c|c|c|c|c|c|c|c|}
\hline & & \multicolumn{3}{|c|}{6} & \multicolumn{3}{|c|}{ Ipsi } \\
\hline & & $\varepsilon_{p}$ & $\varepsilon_{p, l}$ & $\overline{\varepsilon_{p, r}}$ & $\varepsilon_{p}$ & $\varepsilon_{p, l}$ & $\varepsilon_{p, r}$ \\
\hline \multirow{3}{*}{ IHM } & $O x$ & 23.81 & 26.85 & 21.20 & 14.35 & 19.22 & 9.79 \\
\hline & $O y$ & 24.85 & 27.72 & 22.28 & 10.22 & 11.03 & 9.27 \\
\hline & $O z$ & 29.71 & 33.00 & 25.88 & 14.68 & 18.37 & 10.13 \\
\hline \multirow{3}{*}{ OSM } & $O x$ & 21.41 & 23.73 & 18.85 & 6.95 & 11.32 & 3.91 \\
\hline & $O y$ & 18.48 & 18.06 & 19.06 & 6.33 & 7.97 & 4.72 \\
\hline & $O z$ & 21.46 & 22.65 & 19.91 & 7.76 & 12.26 & 5.40 \\
\hline & & \multicolumn{3}{|c|}{ Contra } & \multicolumn{3}{|c|}{ All } \\
\hline \multirow{3}{*}{ IHM } & $O x$ & 36.41 & 32.27 & 48.97 & 13.42 & 16.47 & 11.04 \\
\hline & $O y$ & 30.03 & 23.73 & 41.27 & 10.16 & 10.63 & 9.23 \\
\hline & $O z$ & 40.33 & 36.38 & 46.02 & 18.62 & 27.23 & 12.18 \\
\hline \multirow{3}{*}{ OSM } & $O x$ & 11.36 & 7.30 & 25.84 & 4.66 & 5.36 & 3.92 \\
\hline & $O y$ & 16.83 & 12.27 & 28.65 & 6.00 & 6.15 & 5.84 \\
\hline & $O z$ & 13.70 & 10.44 & 24.75 & 7.78 & 10.38 & 5.53 \\
\hline
\end{tabular}

Table 1: Median position errors (in $\mathrm{mm}$ ) for the 506 dipoles equally distributed in the grey matter as a function of the employed model, dipole orientation, sensor configuration and hemisphere (the indices $l$ and $r$ indicate median errors computed over the left, respectively right hemisphere)

(i) In the case of the 6-sensors configuration, the medians of the localization performance (see table 1) are around $2.5 \mathrm{~cm}$ when using the IHM model and around $2 \mathrm{~cm}$ when using the one sphere model, whatever the considered dipoles orientation and hemisphere (see fig. 3). This is a first confirmation of the modelling superiority of the OSM over the IHM (the same conclusion can be drawn in fact for the four sensor configurations).

The boxplots in figure 3 show that a high localization disparity is obtained for this sensor configuration, which suggests that the relative position of the chosen sensors with respect to the dipole to be localized might be very critical. Indeed, it has to be noticed that better performances can be obtained by using others subset of sensors, raising the issue of the sensors selection.

By studying more carefully the locations of the dipoles that are badly localized using the all-sensors configuration, we observe that the vast majority of these dipoles are located very near to the brain outer limits. This remark is concordant with the considerations given in the section 3.1.

(ii) The localization performances for the ipsilateral-sensors configuration are also confident when using the OSM inversion, clearly better than the IHM. The dipoles situated in the right hemisphere, containing 9 electrodes (i.e. 86 sensors), are better localized than those in the left hemisphere, implanted with 3 electrodes only (26 sensors). Indeed, the mean localization error equals $4.7 \mathrm{~mm}$ at the right, compared to $10.5 \mathrm{~mm}$ at the left (see also fig. 4(a)\&(b), column 2).

(iii) The results obtained using the Contralateral-sensors configuration clearly point out that, in this ideal no-noise case, satisfactory localization performance of a contralateral dipole can be obtained when enough electrodes are implanted in the opposite hemisphere. Indeed, the mean error over the three directions equals $10 \mathrm{~mm}$ for the dipoles located in the left part of the brain, thus localized using the 9 electrodes implanted in the right hemisphere (the results are similar to those obtained using ipsilateral-sensor configuration for this hemisphere). On the other hand, when a limited number of electrodes is available, the localization performance decreases fast: the 


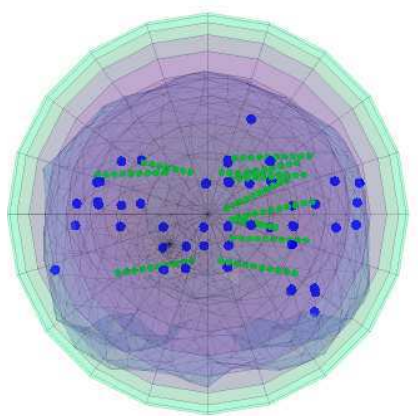

(a)

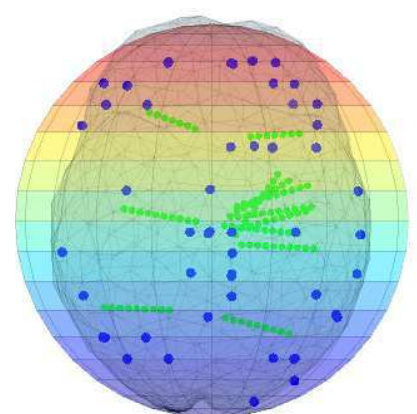

(b)

Figure 5: Positions of the 50 tested dipoles for the noisy simulations within a coarse version of the brain volume mesh. Front view (a) and top view (b). Electrode contacts are figured in green. The OSM used for the localization is superimposed.

mean error value is over $20 \mathrm{~mm}$ when the dipoles of the right hemisphere are localized using the three electrodes of the left hemisphere, and a high disparity can be observed (see figures 3 and 4).

(iv) As expected in the ideal case, the all-sensors configuration gives very confident results. From the table 1, it can be observed that the localization error is around $6 \mathrm{~mm}$ on average for the one sphere model, while it is over $1 \mathrm{~cm}$ for the IHM. From the boxplots (fig.3), it can be seen that the error disparity is rather small, almost $75 \%$ of the dipoles being localized below a $1 \mathrm{~cm}$ error. It has to be noticed that the results are better for the dipoles that are placed in the more implanted (right) hemisphere (mean error $5.1 \mathrm{~mm}$, compared to $7.3 \mathrm{~mm}$ at the left). On the other hand, these right hemisphere dipoles are even better localized using the ipsilateral-sensors configuration $(4.7 \mathrm{~mm}$ average error, see above). This observation seems to indicate that using a sufficient amount of close sensors is better than using all available sensors, even when no noise is present.

\subsubsection{Independent background activities}

As mentioned, the independent background activities are simulated as a spatially and temporally white noise. The 50 selected dipoles (see section 2.1.2) are distributed all over the brain volume and are all accurately localized (position error below 10mm) in the absence of noise by at least one of the two best sensor configurations, i.e., by the all-sensors or by the ipsilateral-sensors configuration. Their positions are given in the figure 5. As seen previously, the OSM overpasses the IHM in all four sensors configurations. The influence of an independent background activity and of a nuisance dipole on the localization performances will be thus analysed using only the OSM.

Table 2 gives a global idea (for the whole head volume and in each hemisphere) on how the noise impacts the localization task for the chosen dipoles. A first analysis globally confirms the no-noise results: all-sensors configuration slightly outperforms, in median, the ipsilateral configuration, while for the specific case of the right hemisphere, the ipsilateral configuration gives more accurate results. The results based on 


\begin{tabular}{|c|c|c|c|c|c|c|}
\hline \multirow{2}{*}{} & \multicolumn{3}{|c|}{ 6 } & \multicolumn{3}{c|}{ Ipsi } \\
\cline { 2 - 7 } & $\varepsilon_{p}$ & $\varepsilon_{p, l}$ & $\varepsilon_{p, r}$ & $\varepsilon_{p}$ & $\varepsilon_{p, l}$ & $\varepsilon_{p, r}$ \\
\hline 0 & 29.3 & 25.4 & 29.8 & 9.2 & 13 & 6.3 \\
\hline$\sigma_{1}$ & 30.3 & 32 & 29.8 & 11.4 & 15.8 & 7.3 \\
\hline$\sigma_{2}$ & 42.2 & 39.8 & 42.9 & 26.3 & 28.1 & 21.1 \\
\hline \multirow{3}{*}{} & \multicolumn{3}{|c|}{ Contra } & \multicolumn{4}{c|}{ All } \\
\cline { 2 - 7 } & $\varepsilon_{p}$ & $\varepsilon_{p, l}$ & $\varepsilon_{p, r}$ & $\varepsilon_{p}$ & $\varepsilon_{p, l}$ & $\varepsilon_{p, r}$ \\
\hline 0 & 23.4 & 13.3 & 28.4 & 7.5 & 6.6 & 7.9 \\
\hline$\sigma_{1}$ & 40.2 & 27.3 & 62.6 & 9.5 & 9.2 & 9.9 \\
\hline$\sigma_{2}$ & 79.5 & 73.7 & 82.6 & 19 & 17.3 & 20 \\
\hline
\end{tabular}

Table 2: Median of the average position errors for the 50 tested dipoles computed over the 100 noise realization $\left(\sigma_{1}\right.$ or $\left.\sigma_{2}\right)$ and over the three directions $O x, O y, O z$, for the four sensor configurations. The results are given for the whole head volume ( $\varepsilon_{p}$ columns), but also with respect to the hemisphere of the dipole $\left(\varepsilon_{p, l}\right.$ (left) and $\varepsilon_{p, r}$ (right) columns). The values in the 0 noise lines are obtained for no noise simulations (they should be similar to those in table 1, except that they are obtained on 50 dipoles instead of 506 )

the contralateral-sensor configuration are highly impacted by the addition of noise, with errors going from about $27 \mathrm{~mm}$ to $83 \mathrm{~mm}$. As expected, the 6-sensors configuration does not provide reliable results, with errors above $30 \mathrm{~mm}$ regardless of the noise power. It is also clear that, in median, the $\sigma_{2}$ noise level is too high to allow good localizations, as all median errors are greater than $10 \mathrm{~mm}$, regardless of the sensor configuration.

Although not shown here, it has to be emphasized that the spans are high, and in both the ipsilateral-sensors and the all-sensors cases we can find very good localizations (with a slight advantage for the ipsilateral configuration when considering the minimum errors). A deeper analysis can be done by considering the positions of the 50 dipoles with respect to the sensors. For conciseness, only the most important observations is given here, emphasizing the importance of the sensors configuration with regard to the dipole position: in both configurations, only the dipoles situated at at most $30 \mathrm{~mm}$ from the closest sensor are correctly localized (especially in the $\sigma_{2}$ noise case), i.e., within an error below $10 \mathrm{~mm}$. Inversely, the most superficial dipoles, situated near the skull border and not surrounded by sensors are badly localized in most cases, even in the $\sigma_{1}$ noise case.

\subsubsection{Physiological nuisance source}

As explained in section 2.1.2, three dipolar sources of interest (D1, D2 and D3) have been simulated, perturbed by another dipole situated in the opposite hemisphere. The results are summarized in table 3 . Considering the results with the additive white noise, only the all-sensors and the ipsilateral-sensors configurations have been tested.

Although the simulations presented in this section are far from being complete, they illustrate the plausible physiological situation when a secondary source is active in a different brain region. As it can be seen from these examples, the no-noise results are consistent with those obtained on the whole set of 506 dipoles: all-sensors configuration is similar to the ipsilateral configuration and the performances are better in the right hemisphere (more implanted) for both configurations. When the nuisance is present, the two configurations continue to provide similar results in the right hemi- 


\begin{tabular}{|c|c|c|c|c|c|c|c|c|c|c|c|c|c|}
\hline & \multicolumn{6}{|c|}{ Ipsi } & \multicolumn{6}{|c|}{ All } \\
\hline & & \multicolumn{3}{|c|}{$\mathrm{L}$} & \multicolumn{3}{|c|}{$\mathrm{R}$} & \multicolumn{3}{|c|}{$\mathrm{L}$} & \multicolumn{3}{|c|}{$\mathrm{R}$} \\
\hline & & D1 & D2 & D3 & D1 & $\overline{\mathrm{D} 2}$ & D3 & D1 & D2 & D3 & $\overline{\mathrm{D} 1}$ & D2 & D3 \\
\hline \multirow{3}{*}{$\mathrm{P}$} & $\overline{0}$ & 5.2 & 5.8 & 4.9 & 1.4 & 1.1 & 2.9 & 5.4 & 2.7 & 5.4 & 1.8 & 1 & 2.9 \\
\hline & $\sigma_{1}$ & 4.5 & 4.7 & 4.8 & 1.9 & 5 & 2.8 & 6.6 & 7.3 & 24.2 & 2.2 & 1.3 & 2.9 \\
\hline & $\sigma_{2}$ & 8 & 15.2 & 13.7 & 3.5 & 1.8 & 2.8 & 53.2 & 85.5 & 101.8 & 4.3 & 2.3 & 23.9 \\
\hline \multirow{3}{*}{$\mathrm{O}$} & 0 & 1.9 & 3.3 & 1.4 & 1.2 & 0.8 & 2.5 & 3.3 & 1.6 & 1.9 & 1.5 & 5.3 & 3.7 \\
\hline & $\sigma_{1}$ & 2.8 & 4.1 & 1.9 & 1.7 & 0.9 & 2.5 & 2.9 & 14.2 & 5.5 & 1.6 & 0.9 & 2.8 \\
\hline & $\sigma_{2}$ & 6.2 & 6.2 & 4.7 & 3.5 & 1 & 2.5 & 5.8 & 68.7 & 23.7 & 2.8 & 5.2 & 2.4 \\
\hline
\end{tabular}

Table 3: Position errors, in $\mathrm{mm}$, for the three tested dipoles in the ideal no-noise case and in the presence of a dipolar nuisance with different amplitudes $\left(\sigma_{1}\right.$ and $\left.\sigma_{2}\right)$. The dipoles to be localized are placed either in the right hemisphere (R) - the most implanted (9 electrodes), or in the left one (L) - less implanted (3 electrodes). The perturbation is placed in the middle of the opposite hemisphere. The two dipoles are either parallel (P) or pointing to the contralateral hemisphere $(\mathrm{O})$.

sphere, better implanted. However one exception is to be noticed for the external dipole D3 in the parallel case with a strong nuisance source $\sigma_{2}$, for which the all-sensors configuration fails (23.9mm error). The performances are poorer in the left hemisphere for both configurations, but almost all the times better for the ipsilateral one. In general, the performances degrade when the depth of the source diminishes and when the amplitude of the perturbation increases but, remarkably, this degradation affects more the all-sensors configuration in the left hemisphere.

\subsection{Localization results on real signals}

\subsubsection{ICS localization}

A first observation is that, although the ICS source is strong, it cannot strictly be considered as dominant with respect to the physiological activity recorded by distant sensors. Indeed the amplitudes of the potentials generated by the ICS decrease very rapidly with the distance and thus the SNR in far electrodes rapidly becomes negative. An example is given figure 6, which illustrates the amplitude of the potentials generated by the ICS dipoles for the three stimulation sites (TB'2-3, TB'4-5 and TB'7-8). As it can be seen, even if the potentials due to the ICS source are visible on all the contacts of the stimulation electrode, their amplitude is very small on the farthest ones. That is, even in the direction of the dipole, the attenuation is very rapid: the potentials at more than $20 \mathrm{~mm}$ have roughly the same magnitude as the background activity. In practice, the ICS signal is visible on (almost all of) the electrodes of the stimulated hemisphere (A', B', TB' and T'), but almost invisible in the contralateral hemisphere, even on the most profound contacts (although this low SNR can be improved by averaging in practical applications).

Having these general considerations in mind, we have tested our localization procedure for three electrical stimulation sites. All of them are situated in the left hemisphere (less implanted), on the same electrode but at different depths inside the brain. The goal was to validate the conclusions of the simulation part and to assess the feasibility of dipole source localization on real SEEG recordings. 

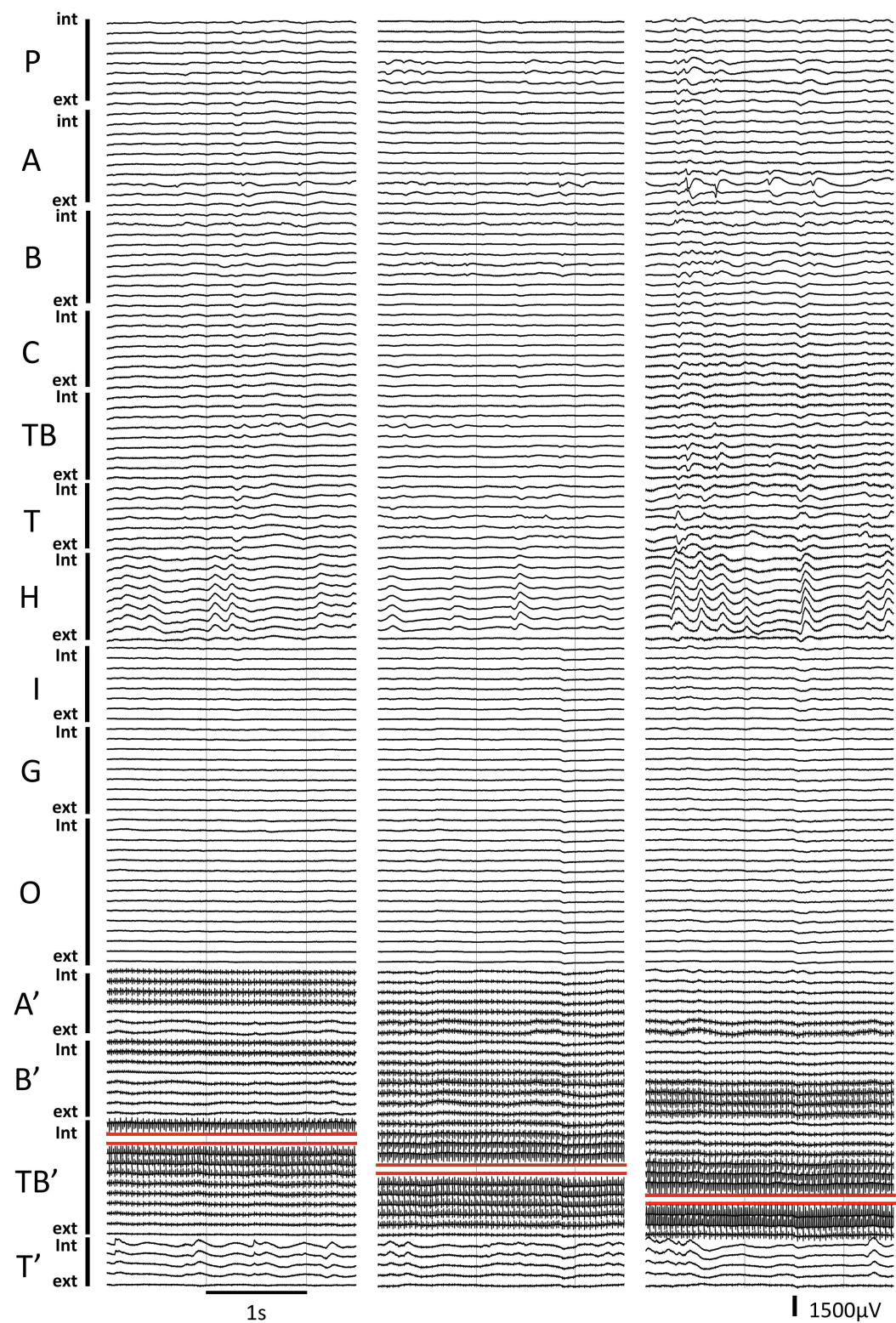

Figure 6: Two and a half seconds of SEEG signals recorded during three ICS sessions. From left to right, the ICS was applied between the TB'2-3, TB'4-5 and TB'7-8 contacts (profound, intermediate, superficial). The stimulation contacts are figured in red 


\begin{tabular}{|l|c|rrr|rrr|rrr|}
\hline \multicolumn{2}{|c|}{} & \multicolumn{3}{|c|}{ TB'2-TB'3 } & \multicolumn{3}{c|}{ TB'4-TB'5 } & \multicolumn{3}{c|}{ TB'8-TB'9 } \\
\hline Cfg & Model & $\varepsilon_{p}$ & $\varepsilon_{a}$ & $G O F$ & $\varepsilon_{p}$ & $\varepsilon_{a}$ & $G O F$ & $\varepsilon_{p}$ & $\varepsilon_{a}$ & $G O F$ \\
\hline 6 & IHM & 21.7 & 48.4 & 100 & 26.9 & 33.7 & 100 & 469.5 & 176.1 & 97.8 \\
& OSM & 18.5 & 46.9 & 100 & 16.2 & 45.8 & 100 & 22.3 & 160.6 & 100 \\
\hline Ipsi & IHM & 2.5 & 8.1 & 99.4 & 4.9 & 19.2 & 99.4 & 1.9 & 22.4 & 99.9 \\
& OSM & 1.0 & 9.0 & 99.6 & 1.3 & 7.4 & 99.8 & 8.9 & 19.5 & 98.6 \\
\hline Contra & IHM & 41.1 & 53.3 & 85.9 & 225.6 & 75.8 & 95.3 & 153.3 & 100.3 & 92.7 \\
& OSM & 40.1 & 74.7 & 88.4 & 105.4 & 115.8 & 95.3 & 88.3 & 93.4 & 92.4 \\
\hline All & IHM & 2.3 & 9.0 & 98.1 & 8.9 & 21.4 & 94.8 & 200.8 & 92.5 & 72.4 \\
& OSM & 0.4 & 9.1 & 97.8 & 3.0 & 14.1 & 97.6 & 92.1 & 86.1 & 73.8 \\
\hline
\end{tabular}

Table 4: Localization results for the three tested ICS dipoles, for all sensors configurations and for both simple propagation models (IHM and OSM). The position error $\varepsilon_{p}$ is given in mm, the orientation error $\varepsilon_{a}$ in degrees and the goodness of fit (GOF) in percents.

The localization was performed with the 4 same sensor configurations as described before: the 6 biggest amplitudes, all available contacts (except those situated on the same electrode as the ICS, namely TB'), all contacts in the ipsilateral hemisphere (again except TB') and all contacts in the contralateral hemisphere. Both IHM and OSM models were used in the inversion procedure. The localization results, in terms of position errors, orientation errors and GOF are given in table 4. Figure 7 allows the visualization of these results.

Analysing table 4, we conclude that the ipsilateral configuration yields the best results, with position errors below $9 \mathrm{~mm}$ and orientation errors below $22.4^{\circ}$ regardless of the dipole position and on the model used for the inversion (IHM or OSM).

If we focus on the profound and intermediate dipoles, the all-sensors configuration also provides good results (below $10 \mathrm{~mm}$ ), but the outer dipole is badly localized.

As in simulation, the contralateral configuration is particularly unreliable (although it has high GOFs). The configuration using the 6 biggest absolute values also leads to poor results (position errors over 16mm), although better choices for the 6 sensors might increase significantly the precision. It has to be noted that the simple 6 sensors setup is particularly sensitive to the performance criterion based on the GOF. Indeed, the GOF is not relevant for this configuration, as it peaks to $100 \%$ for almost all dipoles, regardless of the position errors.

In general, we can conclude that the ipsilateral configuration offers good localization performances regardless of the ICS source position. It confirms the robustness obtained in simulation. The all-sensor configurations shows its limits when considering the outermost ICS source. This result is not necessarily unexpected and it also confirms the simulation results: in real situations, other (unknown) sources are active in the brain, superimposing to the propagation of interest a coloured nuisance on the measurements. Thus the initial hypothesis of one dominant source might not be valid anymore, especially when the ICS dipole is far from the center of the head and is thus hardly visible to the electrodes situated in the opposite hemisphere (see also the 6-channels SEEG example presented in subsection 2.1.3).

\subsubsection{Epileptic spikes localization}

We present here an example of epileptic spikes localization. The results on this example are very convincing (see figure 8): after eliminating the electrode on which the 


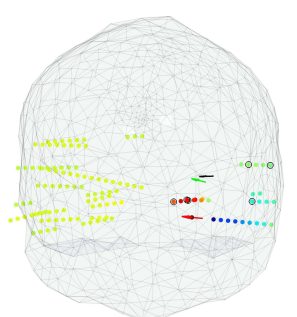

(a)

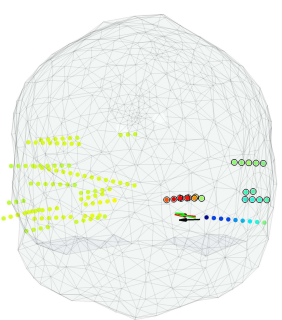

(d)

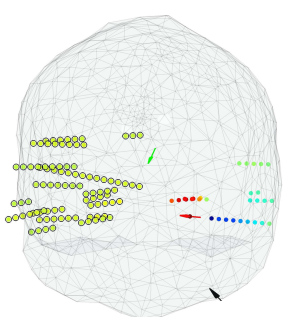

(g)

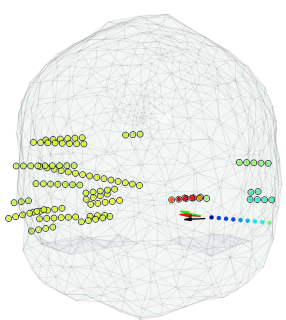

(j)

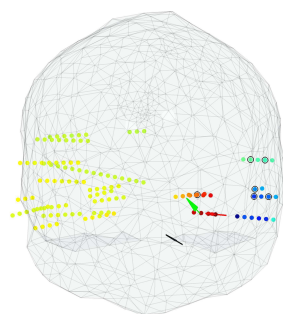

(b)

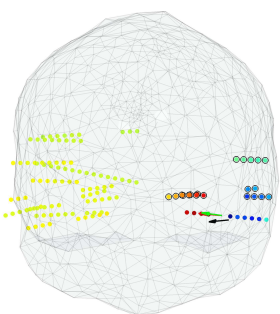

(e)

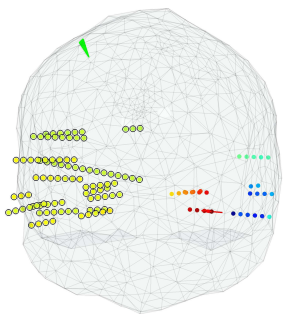

(h)

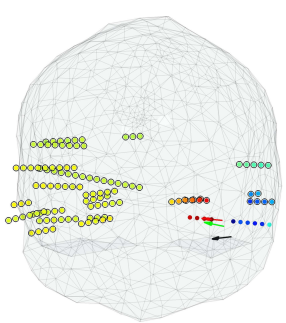

(k)

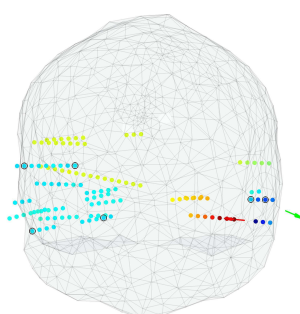

(c)

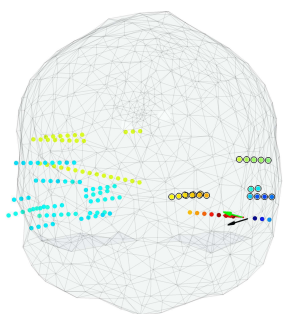

(f)

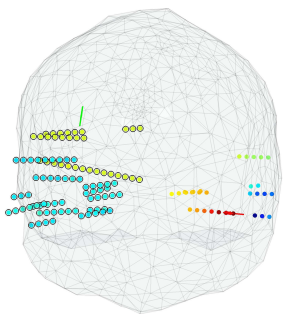

(i)

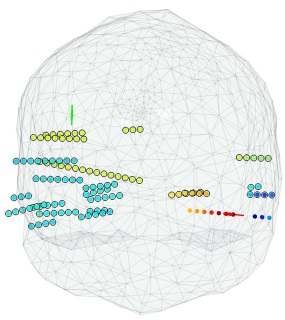

(1)

Figure 7: Localization results for the ICS dipoles situated between TB'2-3 (first column), TB'4-5 (second column) and TB'8-9 (third column) : (a,b,c) using only 6 contacts, with the biggest measured signals in absolute values; (d,e,f) using all contacts in the ipsilateral hemisphere, except those of the stimulation electrode; $(\mathrm{g}, \mathrm{h}, \mathrm{i})$ using all contacts in the contralateral hemisphere; $(\mathrm{j}, \mathrm{k}, \mathrm{l})$ using all contacts, except those belonging to the stimulation electrode. The color scale on each contact represents the time-averaged value of the recorded potential (from dark red for highest values to dark blue for lowest values). The used contacts are circled in black on all figures. The red arrow indicates the ICS dipole position and orientation, the black arrow indicates the results of the localization procedure using the IHM and the green arrow the dipole estimated using the OSM. The IHM results (black arrow) is missing in figures (c,h,i,l) as the estimated dipoles converged outside the head volume. The actual values of the errors and the corresponding GOF are given in the table 4 

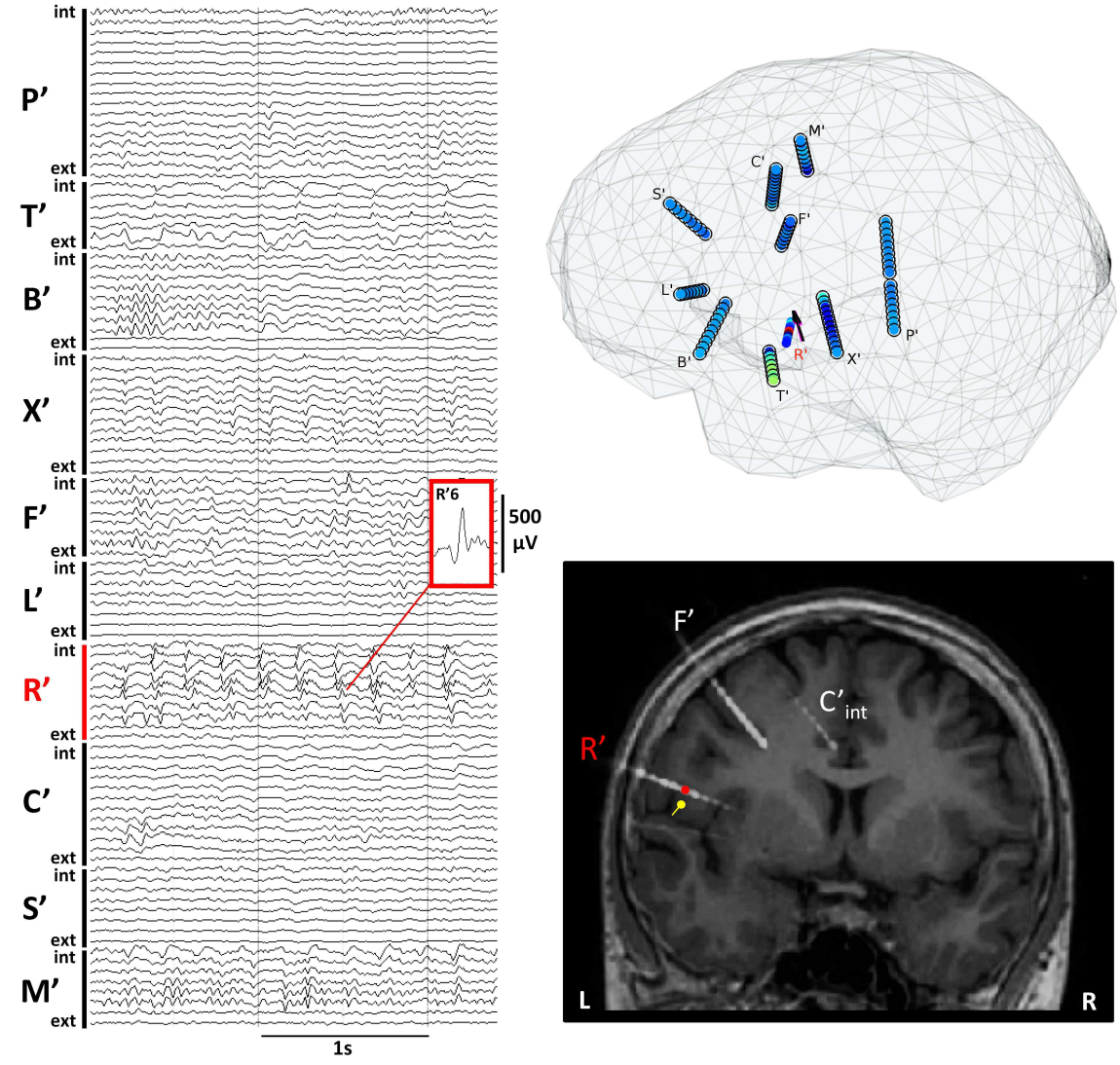

$\mu \mathrm{V}$

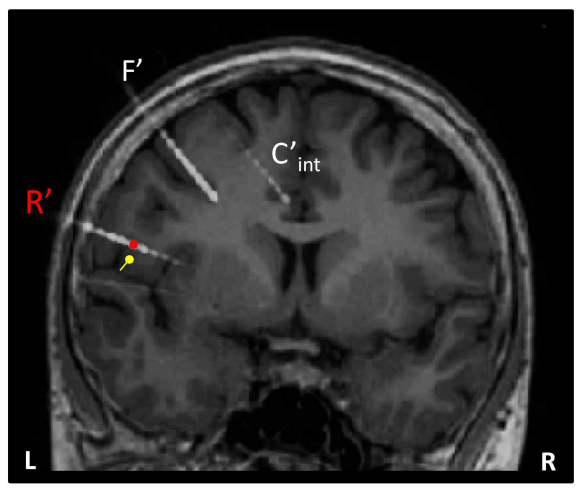

Figure 8: Source localization of interictal epileptic spikes: case of a 28 year-old women with drug-resistant epilepsy. Left side: SEEG signals in common reference montage (FPz scalp electrode) during interictal period. Epileptic spikes were recorded in the middle contacts of R' depth electrode (left central operculum). Top right: 3D view of all depth electrodes in a realistic brain mesh. The sensors used for localization are circled in black. The localized dipoles, almost superimposed, are figured in black (IHM) and in magenta (OSM) (coordinates of the origin at $\mathrm{x}:-50.1 \mathrm{~mm}$; $\mathrm{y}:-4.8 \mathrm{~mm}$; $\mathrm{z}: 27.2 \mathrm{~mm}$ ). Bottom right: frontal slice of CT-MR co-registration that shows trajectories of depth electrodes R', F' and a part of C'. Red dot indicates the position (x: $-47.5 \mathrm{~mm} ; \mathrm{y}:-6.3 \mathrm{~mm} ; \mathrm{z}: 30.7 \mathrm{~mm})$ of R' 6 contact where epileptic spikes were recorded with the highest amplitude. The dipole source (OSM inversion in yellow) was localized in the left central operculum at $4.6 \mathrm{~mm}$ distance from R' 6 . 
spikes present a maximum amplitude ( $\left.\mathrm{R}^{\prime}\right)$, the localization was made using both analytical models (IHM and OSM), after having selected and averaged over 20 spikes using the automatic procedure explained in section 2.1.4. The results are very similar and very precise: the source is localized at less than $5 \mathrm{~mm}$ from the contact presenting the highest amplitude (R'6), in the same anatomical structure (left central operculum). The obtained GOF is correct ( $72 \%$ for IHM and 74\% for OSM), knowing that other sources might be present in the distant explored structures. The results above are confirmed when localizing using all available sensors, including those from the R' electrode. In this case, the distance between R' 6 and the origin of the dipole diminishes to $1.2 \mathrm{~mm}$ for the OSM based inversion and to $1.4 \mathrm{~mm}$ for the IHM, with almost similar GOFs of $77 \%$ for the IHM and $78 \%$ for the OSM.

Interestingly enough, the number of electrodes used for localization can be drastically reduced. Obviously, the results depend on the chosen spatial configuration. For example, when preserving only 3 electrodes (B', T' and X') having 29 contacts, the OSM still provides a localization at $7.5 \mathrm{~mm}$ from R' 6 , while the IHM converges at $6.3 \mathrm{~mm}$, with GOFs equal to $82 \%$ for both models. Last but not least, these good results are coherent among them, with a maximal distance between the solutions below $7 \mathrm{~mm}$ regardless of the forward model and the chosen sensor configuration (among the 3 configurations discussed above).

Finally, we also perform the localization on each of the twenty epileptic spikes separately, using either all available contacts or only the 3 selected electrodes B', T' and $\mathrm{X}^{\prime}$. For each spike, the time samples above the threshold described in section 2.1.4 are retained in the localization process. As expected, the results are less accurate than in the averaged case, illustrating the importance of the averaging pointed out in the section 2. For the first sensor configuration (all contacts in the hemisphere, except those on R'), the mean position of these twenty estimated dipoles is placed at $13.2 \mathrm{~mm}$ from R'6, with standard deviations of 18.7, 18.0 and 23.0mm in $x, y$ and $z$ directions respectively. For the second configuration (29 sensors only, on 3 electrodes), the mean position is at $9.2 \mathrm{~mm}$, with standard deviations of $14.1,6.5$ and $5.9 \mathrm{~mm}$ respectively. It is important to notice that using only the sensors having a rather high SNR (close to the presumed origin of the spike) improves the quality of individual spikes localization, as the time averaging becomes less critical.

\section{Discussion}

SEEG is of great interest when non-invasive recordings give inconclusive results. In clinical situation, real SEEG measurements are commonly used in bipolar montages (Bartolomei et al., 2008; Maillard et al., 2009), because they offer a focal view of the electrical activity of brain regions of interest by cancelling remote activities. In this study, we use the fact that strong brain electrical generators propagate by volume conduction and so can be recorded by SEEG electrodes observed in a common reference montage.

Considering the environment models, there is a vast choice, both for analytical and numerical ones. Because of the spatial distribution of the sensors (close to the epileptic foci and relatively far from the skull), we assumed that the use of analytic propagation 
models (namely IHM or OSM) might provide sufficient modelling accuracy. According to the forward model comparison presented in (Cosandier-Rimélé et al., 2007), the difference between a three-layer head model and the IHM model is rather small. These observations are consistent with our findings that the difference between respectively the IHM model and the OSM with respect to the FEM based five-layer homogeneous isotropic model is rather small when considering the profound areas of the brain volume (see the section 3.1).

The aim of this paper is to analyse the feasibility of a straightforward localization method based on a single dipole using both an infinite homogeneous medium (IHM) and a one-sphere model (OSM).We illustrate our study using the ICS signal, as well as on an example of real epileptic spikes.

For solving the localization inverse problem, we have chosen a modified version of the Scherg's ECD approach, already validated for epileptic patterns in (Gavaret et al., 2009; Koessler et al., 2010). A multi-start strategy is applied using 19 initializations (well distributed in the brain volume), and the chosen solution is the one maximizing the goodness of fit (GOF) criterion (equation 9). We have tested the proposed approach by extensive simulations and on real examples.

Analysing the localization results obtained in the simulated no-noise setup, a first global conclusion that can be drawn from table 1 is that the OSM is, in median, more accurate than the IHM, regardless of the sensor configuration and the direction of the dipoles. Conclusions regarding the best sensor configuration are less obvious. If it is clear that the 6 -sensors configuration is the worse ${ }^{4}$, followed by the contralateral configuration, it is not obvious to choose between the ipsilateral and the all-sensors configurations. Indeed, even if the all-sensors configuration is the best option globally, i.e., regardless of the hemisphere, the ipsilateral is slightly better in the right (more implanted) hemisphere. Moreover, considering either the ipsilateral or the all-sensors configuration, the sources implanted in this particular right hemisphere are better localized than those implanted in the left hemisphere. These observations suggest that, even in the absence of noise, the quality of localization is determined by two important factors: using as many as possible and as close as possible electrodes to the presumed dipole localization gives more reliable results (see also figure 3 ).

We can conclude that if the lateralization of the researched dipole is known, it can be accurately localized using only ipsilateral sensors, with median errors around $5 \mathrm{~mm}$ if their number is high (right hemisphere, $\varepsilon_{p, r}$ for the ipsilateral based localization using the OSM) and about $10 \mathrm{~mm}$ if they are rather sparsely implanted (left hemisphere, $\left.\varepsilon_{p, l}\right)$. On the other hand, even if the localization precision seems globally better when using all electrodes, the improvement might not be sufficient to justify, in real cases, the implantation of a great number of electrodes. However, it is necessary to compare more extensively the proposed configurations in order to assess the statistical validity of the differences pointed out by the medians in table 1 .

The position and orientation of the dipoles with respect to the electrodes has also a certain influence. Although not noticeable from the table, profound dipoles are gener-

\footnotetext{
${ }^{4}$ This observation must be nuanced because of the automatic choice of the 6 sensors: much better results can be obtained by manual selection.
} 
ally better localized than the ones close to the brain limit. This can be seen for example in figures $4(\mathrm{a}) \&(\mathrm{~b})$. Several explanations are plausible: model errors are higher when approaching tissue limits; fewer sensors record a sufficiently high signal because of the distance. The impact of the orientation is also visible. For example, for $O x$ and $\mathrm{Oz}$ oriented dipoles having origins outside the volume surrounded by the electrodes, the results are not satisfactory. This can be explained by the fact that the electrodes are implanted nearly orthogonally on the $z O x$ plane, mostly in the bottom area of the brain (the targeted structures being situated in the temporal lobe). Thus all the dipoles placed in the upper or lower part of the brain, as well as those situated very close to the frontal and occipital areas, are seen as monopoles by the electrodes (only positive or only negative values being recorded), bringing less variety in the measurements and yielding poorer performances.

These observations are confirmed by the additive white noise simulations. Such noise allows to analyse ill-conditioned configurations where weaknesses of the localization method are revealed. With a low noise level ( $\sigma_{1}$ noise level in our simulation), many dipoles located out of the volume defined by the electrodes (i.e., in the upper and lower part of the brain and near the outer limits of the brain) are poorly localized, and none of these outer dipoles are retrieved when the additive noise is stronger $\left(\sigma_{2}\right)$. In this last case, a good reconstruction is obtained only for dipoles that are in the cloud of sensors and located sufficiently close to a sensor (under $30 \mathrm{~mm}$ ), thus collecting a good SNR for this source. Globally, the method is expected to give reliable results when considering deep to intermediate dipoles with enough surrounding sensors and with favourable SNR $\left(\sigma_{1}\right.$, e.g. when the averaging is done on a large number of time instants). When the source is weak or/and with few trials available for averaging $\left(\sigma_{2}\right.$ case), the validity of the result is questionable.

The addition of a parasite dipole emphasizes further the importance of the sensor configuration. Indeed, the ipsilateral shows more robustness to the spurious activity; this is not unexpected, as the dominant source hypothesis is severely challenged by the presence of the nuisance, which is far more disruptive than the white noise, and this especially for the sensors far from the source to be localized. Globally, the localization based on the ipsilateral-sensor configuration provides similar or better performances than the all-sensor configuration, showing that an adequate sensor selection avoiding the less relevant measurements enhances the localization performance. In practice, keeping the sensors within the ipsilateral hemisphere implies keeping the measurements with the highest SNR and thus less impacted by possible disruptive activity in the opposite hemisphere.

Our tests on real ICS-SEEG data confirm these observations, especially for the configuration of sensors to be retained for the localization. A first observation is that, as for the simulated dipoles, the OSM based localization is generally more accurate than IHM based one. From a configuration point of view, the most efficient is confirmed to be the ipsilateral, i.e., the best choice is to use the sensors situated in the hemisphere (thus in the neighbourhood) of the dipole. The all-sensors configuration is less accurate, especially for the most external dipole. An inspection of the SEEG traces of the opposite hemisphere gives evidence on the presence of nuisance sources, explaining this decrease in performance as illustrated on simulation.

In these three examples, the results are particularly significant. Indeed, we deal 
here with the less-implanted hemisphere, and we might then go further and conclude that a low number of well implanted electrodes in the suspected hemisphere (i.e., close enough from the source) are sufficient for precise localization. Moreover, for this configuration involving 19 sensors, the GOF is high and it is representative for the localization precision. From our experiments on simulated signal as well as on ICS signals, selecting the sensors of the ipsilateral-hemisphere seems to stand for a first valid recommendation when dealing with the localization of a single dominant dipole.

Finally, we have tested the proposed method on epileptic spikes, with the aim to demonstrate that dipolar source localization is indeed possible on real physiological signals using SEEG measurements. In such situation, the SNR is more typical to a physiological context than in the highly energetic ICS case. Our source localization, implemented using several configurations of depth electrodes, gives consistent localization with the irritative zone defined by SEEG. For this patient there is a perfect anatomical concordance (left central operculum) between the lesional zone (MR lesion), irritative zone (interictal epileptic spikes) and epileptogenic zone (epileptic seizure). We can conclude that electrical source localization can accurately localize focal epileptic source with distant depth EEG electrodes (we have removed the depth electrode that recorded the epileptic spikes with the highest amplitude). For this particular patient, SEEG-guided radiofrequency thermolesions (Guénot et al., 2004) of the left central operculum was done at the end of the SEEG investigation. The patient is seizure free since the intervention, 3 years ago, confirming thus the precise and correct localization of the SEEG investigation and therefore of our source localization.

The global conclusion of these simulations and real examples is that using a sufficient amount of close electrodes is the best option for accurate source localization. Time averaging seems to be unavoidable for reliable localization, although the number of necessary time samples depend on the spatial configuration of the employed sensors. In particular, using only ipsilateral sensors (and not necessarily a very high number of sensors) consistently yields dipole localization within $10 \mathrm{~mm}$ from the origin. In clinical situations, it is not a strong constraint nor a difficult choice to make. Indeed, privileging one hemisphere is a frequent situation because in most of the cases, non-invasive presurgical investigations allow lateralizating the epileptogenic zone with a high degree of confidence (Caparos et al., 2006; Cecchin et al., 2010). In the rare ambiguous cases the number of depth electrodes is balanced between the two hemispheres.

There are several limitations to our approach. In this paper, we argue that selecting the sensors close to the region of interest is preferable than considering the whole set of sensors. This rough conclusion regarding the sensor configuration when facing disruptive activities will have to be refined. Two main directions will be considered in future research: (i) looking for a subset of geometrically well positioned sensors or (ii) considering the simultaneous localization of multiple sources. The former would correspond to an automated or semi-automated subgrouping of the sensors to perform the localization of a dominant source in the targeted area, other activities being considered as noise. The latter would correspond to the hypothesis that, instead of one dominant activity, we have a finite amount of dominant activities (several dominant sources (Chang et al., 2005)), which have to be localized simultaneously. One have to mention that, in the case of multiple sources, the least-square approaches might cause (more) undesired local minima problems, advanced localization methods have to be 
developed in such situations (e.g. (Kiebel et al., 2008)).

It has to be noticed that the GOF criterion might not be the best choice for choosing among the localizations issued from the multi-start procedure. From our experiments on simulation, the maximum GOF do not always indicate the minimum position error. If the best solutions were indeed chosen, the median of the position errors would be lowered with respect to the one provided by the GOF selected solutions. This is particularly true in the case of the 6-sensors configuration, where we found out that the median values given in the table 1 for the OSM based localization can be lowered by about $1 \mathrm{~cm}$. An interesting development might be the design of a new criterion for choosing among the results returned by the multi-start procedure, more respectful of the position accuracies.

Finally, the enhancement of the forward model has also to be studied in order to take into account sources close to the tissues' frontiers, at least by adapting the spherical model to the local geometry.

In practical applications, the aim of SEEG investigations is to precisely identify and delineate the epileptogenic zone in order to propose a curative surgery. By consequent depth electrodes are placed in these pathological areas and at the edges of them in order to be able to differentiate healthy cortex from the epileptogenic zone. In this context, our method could give valuable information concerning the distribution of the epileptic sources in a larger volume without the need of additional electrodes. Moreover, ESL from intra-cerebral recordings could be an interesting method to investigate in a noninvasive way the brain regions where vascular constraints avoid the placement of depth electrodes.

The approach proposed in this paper needs to be clinically validated on a larger number of real electrophysiological signals like ictal/interictal epileptic patterns or intra-cerebral evoked potentials. These validations will be carried out with the help of neurologists, who will expertise a large SEEG database in order to label different sources configurations with different degrees of conformity to our hypothesis (single dominant/multiple sources, deep/lateral sources...). If accuracy and reliability of electrical source imaging using SEEG signals are confirmed using physiological sources, we intend to identify a standarized implantation of a limited number of electrodes able to localize events in the whole brain volume. To conclude, focal view of bipolar SEEG signals interpretation combined with the global view of the electrophysiological activity using monopolar SEEG signals can really give a complete and precise overview of the local and remote brain sources.

\section{Acknowledgments}

The authors thank Prof. S. Colnat-Coulbois (Nancy) for the neurosurgical contributions and the Regional Council of Lorraine (France), the project PEPS APSE and the project ANR SEPICOT for their financial support.

\section{References}

Baillet, S., Mosher, J., Leahy, R., 2001. Electromagnetic brain mapping. Signal Processing Magazine, IEEE 18 (6), 14-30. 
Bartolomei, F., Chauvel, P., Wendling, F., 2008. Epileptogenicity of brain structures in human temporal lobe epilepsy: a quantified study from intracerebral EEG. Brain 131 (7), 1818-1830.

Caparos, M., Louis-Dorr, V., Wendling, F., Maillard, L., Wolf, D., 2006. Automatic lateralization of temporal lobe epilepsy based on scalp $\{E E G\}$. Clinical Neurophysiology 117 (11), $2414-2423$.

URL http://www.sciencedirect.com/science/article/pii/ S138824570601371X

Caune, V., Le Cam, S., Ranta, R., Maillard, L., Louis-Dorr, V., 2013. Dipolar source localization from intracerebral SEEG recordings. In: Engineering in Medicine and Biology Society (EMBC), 2013 35th Annual International Conference of the IEEE. pp. 41-44.

Cecchin, T., Ranta, R., Caspary, O., Koessler, L., Vespignani, H., Maillard, L., 2010. Seizure lateralization in scalp EEG using Hjorth parameters. Clinical Neurophysiology $121,290-300$.

Chang, N., Gulrajani, R., Gotman, J., 2005. Dipole localization using simulated intracerebral EEG. Clinical Neurophysiology 116 (11), 2707 - 2716.

Cosandier-Rimélé, D., Badier, J.-M., Chauvel, P., Wendling, F., march 2007. A physiologically plausible spatio-temporal model for EEG signals recorded with intracerebral electrodes in human partial epilepsy. Biomedical Engineering, IEEE Transactions on 54 (3), $380-388$.

Dümpelmann, M., Ball, T., Schulze-Bonhage, A., 2012. sLORETA allows reliable distributed source reconstruction based on subdural strip and grid recordings. Human Brain Mapping 33 (5), 1172-1188.

URL http: //dx.doi.org/10.1002/hbm.21276

Dümpelmann, M., Fell, J., Wellmer, J., Urbach, H., Elger, C. E., 2009. 3D source localization derived from subdural strip and grid electrodes: A simulation study. Clinical Neurophysiology 120 (6), 1061 - 1069.

URL http://www.sciencedirect.com/science/article/pii/ S138824570900251X

Gavaret, M., Trébuchon, A., Bartolomei, F., Marquis, P., McGonigal, A., Wendling, F., Regis, J., Badier, J., Chauvel, P., 2009. Source localization of scalp-EEG interictal spikes in posterior cortex epilepsies investigated by HR-EEG and SEEG. Epilepsia 50 (2), 276-289.

Geddes, L., Baker, L., 1967. The specific resistance of biological material - a compendium of data for the biomedical engineer and physiologist. Medical and biological engineering 5 (3), 271-293.

Gharib, S., Sutherling, W., Nakasato, N., Barth, D., Baumgartner, C., Alexopoulos, N., Taylor, S., Rogers, R., 1995. MEG and ECoG localization accuracy test. Electroencephalography and clinical neurophysiology 94 (2), 109-114. 
Greenblatt, R., Ossadtchi, A., Pflieger, M., 2005. Local linear estimators for the bioelectromagnetic inverse problem. IEEE Transactions on Signal Processing 53 (9), 3403-3412.

Guénot, M., Isnard, J., Ryvlin, P., Fischer, C., Mauguière, F., Sindou, M., 2004. SEEGguided RF thermocoagulation of epileptic foci: Feasibility, safety, and preliminary results. Epilepsia 45 (11), 1368-1374.

Hofmanis, J., 2013. Contribution to the cerebral forward model by depth electric stimulation and SEEG measurements : application in epilepsy. Ph.D. thesis, Université de Lorraine, Nancy, Fance.

URL http://tel.archives-ouvertes.fr/tel-00919480

Hofmanis, J., Caspary, O., Louis-Dorr, V., Maillard, L., Jan. 2011. Automatic depth electrode localization in intracranial space. In: 4th International Conference on Bioinspired Systems and Signal Processing, Biosignals 2011. Rome, Italie.

Hofmanis, J., Caspary, O., Louis-Dorr, V., Ranta, R., Maillard, L., 2013. Denoising depth EEG signals during DBS using filtering and subspace decomposition. Biomedical Engineering, IEEE Transactions on 60 (10), 2686-2695.

Hu, S., Stead, M., Worrell, G., Apr. 2008. Removal of scalp reference signal and line noise for intracranial EEGs. In: Networking, Sensing and Control, 2008. ICNSC 2008. IEEE International Conference on. pp. 1486-1491.

Jonas, J., Descoins, M., Koessler, L., Colnat-Coulbois, S., Sauvée, M., Guye, M., Vignal, J.-P., Vespignani, H., Rossion, B., Maillard, L., 2012. Focal electrical intracerebral stimulation of a face-sensitive area causes transient prosopagnosia. Neuroscience $222(0), 281-288$

Jonas, J., Frismand, S., Vignal, J.-P., Colnat-Coulbois, S., Koessler, L., Vespignani, H., Rossion, B., Maillard, L., 2014. Right hemispheric dominance of visual phenomena evoked by intracerebral stimulation of the human visual cortex. Human Brain Mapping, to appear.

URL http://dx.doi.org/10.1002/hbm.22407

Kiebel, S. J., Daunizeau, J., Phillips, C., Friston, K. J., 2008. Variational Bayesian inversion of the equivalent current dipole model in EEG/MEG. NeuroImage 39 (2), $728-741$.

Koessler, L., Salido-Ruiz, R., Ranta, R., Louis-Dorr, V., Gavaret, M., Maillard, L., 2010. Influence of source separation and montage on ictal source localization. In: Engineering in Medicine and Biology Society (EMBC), 2010 Annual International Conference of the IEEE. pp. 2898 -2901.

Madhu, N., Ranta, R., Maillard, L., Koessler, L., 2012. A unified treatment of the reference estimation problem in depth EEG recordings. Med. Biol. Eng. Comput. 50 (10), 1003-1015. 
Maillard, L., Barbeau, E. J., Baumann, C., Koessler, L., Bénar, C., Chauvel, P., Liégeois-Chauvel, C., 2011. From perception to recognition memory: Time course and lateralization of neural substrates of word and abstract picture processing. Journal of Cognitive Neuroscience 23 (4), 782 - 800.

Maillard, L., Koessler, L., Colnat-Coulbois, S., Vignal, J., Louis-Dorr, V., Marie, P., Vespignani, H., 2009. Combined SEEG and source localization study of temporal lobe schizencephaly and polymicrogyria. Clinical Neurophysiology 120, 16281636.

Michel, C., Murray, M., Lantz, G., Gonzalez, S., Spinelli, L., Grave de Peralta, R., 2004. EEG source imaging. Clinical neurophysiology 115 (10), 2195-2222.

Oosterom, A., 2012. The inverse problem of bioelectricity: an evaluation. Medical \& Biological Engineering \& Computing 50 (9), 891-902.

Pascual-Marqui, R. D., Oct. 2007. Discrete, 3D distributed, linear imaging methods of electric neuronal activity. Part 1: exact, zero error localization. arXiv:0710.3341 [math-ph], 2007-October-17, http://arxiv.org/pdf/0710.3341, 1-16.

Plummer, C., Harvey, A. S., Cook, M., 2008. EEG source localization in focal epilepsy: Where are we now? Epilepsia 49 (2), 201-218.

Ramantani, G., Cosandier-Rimélé, D., Schulze-Bonhage, A., Maillard, L., Zentner, J., Dümpelmann, M., 2013. Source reconstruction based on subdural EEG recordings adds to the presurgical evaluation in refractory frontal lobe epilepsy. Clinical Neurophysiology 124 (3), $481-491$.

URL http://www.sciencedirect.com/science/article/pii/ S1388245712005986

Ranta, R., Salido-Ruiz, R., Louis-Dorr, V., Aug. 2010. Reference estimation in EEG recordings. In: 32nd Ann. Int. Conf. of the IEEE-EMBS. Buenos Aires, Argentina.

Scherg, M., 1990. Fundamentals of dipole source potential analysis. Auditory evoked magnetic fields and electric potentials. Advances in audiology 6, 40-69.

Vallaghé, S., 2008. EEG and MEG forward modeling : computation and calibration. Ph.D. thesis, University of Nice - Sophia Antipolis, France.

von Ellenrieder, N., Beltrachini, L., Muravchik, C. H., 2012. Electrode and brain modeling in stereo-EEG. Clinical Neurophysiology to appear.

Yao, D., 2000. Electric potential produced by a dipole in a homogeneous conducting sphere. Biomedical Engineering, IEEE Transactions on 47 (7), 964-966.

Yvert, B., Fischer, C., Bertrand, O., Pernier, J., 2005. Localization of human supratemporal auditory areas from intracerebral auditory evoked potentials using distributed source models. Neuroimage 28 (1), 140-153. 
Zaveri, H., Duckrow, R., Spencer, S., 2009. Concerning the observation of an electrical potential at a distance from an intracranial electrode contact. Clinical Neurophysiology $120,1873-1875$.

Zhang, Y., van Drongelen, W., Kohrman, M., He, B., 2008. Three-dimensional brain current source reconstruction from intra-cranial ECoG recordings. NeuroImage $42(2), 683-695$. 